\title{
Isolated polycystic liver disease genes define effectors of polycystin-1 function
}

\author{
Whitney Besse, ${ }^{1}$ Ke Dong, ${ }^{1}$ Jungmin Choi, ${ }^{2}$ Sohan Punia, ${ }^{1}$ Sorin V. Fedeles, ${ }^{1}$ Murim Choi, ${ }^{2}$ Anna-Rachel Gallagher, ${ }^{1}$ \\ Emily B. Huang, ${ }^{1}$ Ashima Gulati, ${ }^{1}$ James Knight, ${ }^{2}$ Shrikant Mane, ${ }^{2}$ Esa Tahvanainen, ${ }^{3}$ Pia Tahvanainen, ${ }^{3}$ \\ Simone Sanna-Cherchi, ${ }^{4}$ Richard P. Lifton, ${ }^{2}$ Terry Watnick, ${ }^{5}$ York P. Pei, ${ }^{6}$ Vicente E. Torres, ${ }^{7}$ and Stefan Somlo ${ }^{1,2}$ \\ 1Department of Internal Medicine, and 2Department of Genetics, Yale University School of Medicine, New Haven, Connecticut, USA. ${ }^{3}$ Department of Medical Genetics, University of Helsinki, Helsinki, Finland. \\ ${ }^{4}$ Department of Medicine, Columbia University, New York, New York, USA. 'Department of Medicine, University of Maryland School of Medicine, Baltimore, Maryland, USA. ${ }^{6}$ Division of Nephrology, \\ University Health Network, Toronto, Ontario, Canada. 'Division of Nephrology and Hypertension, Mayo Clinic, Rochester, Minnesota, USA.
}

\begin{abstract}
Dominantly inherited isolated polycystic liver disease (PCLD) consists of liver cysts that are radiologically and pathologically identical to those seen in autosomal dominant polycystic kidney disease, but without clinically relevant kidney cysts. The causative genes are known for fewer than $40 \%$ of PCLD index cases. Here, we have used whole exome sequencing in a discovery cohort of 102 unrelated patients who were excluded for mutations in the 2 most common PCLD genes, PRKCSH and SEC63, to identify heterozygous loss-of-function mutations in 3 additional genes, ALC8, CANAB, and SEC61B. Similarly to PRKCSH and SEC63, these genes encode proteins that are integral to the protein biogenesis pathway in the endoplasmic reticulum. We inactivated these candidate genes in cell line models to show that loss of function of each results in defective maturation and trafficking of polycystin-1, the central determinant of cyst pathogenesis. Despite acting in a common pathway, each PCLD gene product demonstrated distinct effects on polycystin-1 biogenesis. We also found enrichment on a genome-wide basis of heterozygous mutations in the autosomal recessive polycystic kidney disease gene PKHD1, indicating that adult PKHD1 carriers can present with clinical PCLD. These findings define genetic and biochemical modulators of polycystin-1 function and provide a more complete definition of the spectrum of dominant human polycystic diseases.
\end{abstract}

\section{Introduction}

Isolated polycystic liver disease (PCLD; also called ADPLD) presents in adult life with radiologically and pathologically identical liver cysts to those seen in autosomal dominant polycystic kidney disease (ADPKD), but without the burden of kidney cysts required for a diagnosis of ADPKD (1-4). Liver cysts in both PCLD and ADPKD are lined with biliary epithelium. Cysts may arise from ductal plate malformations known as von Meyenburg complexes, which result from abnormalities in late embryonic biliary genesis $(2,5,6)$, although animal model data show that liver cysts in ADPKD and PCLD can occur in adult life without developmentally determined biliary tract malformations $(7,8)$. While the inheritance pattern and adult onset of isolated liver cysts in PCLD parallel those of ADPKD, clinical detection may be reduced because liver cysts are often asymptomatic or nonpenetrant $(1,2)$. Symptomatic PCLD results if the liver size becomes large enough to cause mass effects including abdominal distension, pain, early satiety, and, rarely, biliary or portal obstruction. Symptoms tend to be more severe in women $(2,9)$. Infrequently, symptoms can be severe enough to require

Authorship note: J. Choi, S. Punia, and S.V. Fedeles contributed equally to this work. Conflict of interest: The authors have declared that no conflict of interest exists. Submitted: September 27, 2016; Accepted: February 9, 2017 Reference information: / Clin Invest. 2017;127(5):1772-1785 https://doi.org/10.1172/JCI90129. cyst fenestration, partial hepatectomy, or total hepatectomy with liver transplant (10).

Familial linkage-based studies and positional cloning identified autosomal dominant inheritance of loss-of-function mutations in PRKCSH and SEC63 as causing PCLD (1, 11-13). The respective protein products, glucosidase II $\beta$ (GII $\beta$ ) and SEC63, play essential roles in co- and posttranslational modification of membrane and secreted proteins in the endoplasmic reticulum (ER). This was an unexpected localization for polycystic disease gene products since most fibrocystic diseases of the kidney and liver, including ADPKD, are associated with proteins that function in primary cilia (14). Primary cilia are minute solitary membraneenclosed microtubule-based projections that serve as organelles integrating cellular sensory signals on the apical surface of many cell types, including bile duct and kidney tubule epithelial cells (15-17). Mutations in another noncilial protein, LDL receptorrelated protein 5 (LRP5), a coreceptor in canonical Wnt signaling, have also been implicated as causing PCLD (18).

ADPKD, the most common monogenic kidney disease, results in age- and genotype-dependent progression to end-stage renal disease. It is caused by mutations in PKD1 and PKD2, which encode polycystin-1 (PC1) and polycystin-2 (PC2), respectively. PC1 and PC2 are large complex integral membrane proteins that are functionally expressed on primary cilia. PC1 and PC2 are among the many client proteins of GII $\beta$ and SEC63 in the ER protein biogenetic pathway. Although inherited as dominant traits, 
cyst initiation in ADPKD and PCLD is thought to occur following somatic second-hit mutations that result in complete or partial inactivation of the normal allele of the respective disease gene, leading to a recessive genotype in individual cells (7, 19-22). These cells initiate processes that lead to tissue remodeling and cyst formation over the span of decades. It was hypothesized that loss of function in either GII $\beta$ or SEC63 results in impaired biogenesis and maturation of $\mathrm{PC} 1$ and $\mathrm{PC} 2$, and it is this reduction in polycystin protein dosage that results in cyst formation in PCLD (13). This hypothesis was validated in vivo by showing that orthologous gene animal models with conditional inactivation of Sec63 and Prkcsh develop both kidney and liver cysts in a PC1 dosage-dependent manner (7). These studies established that the PCLD genes are modifiers of $\mathrm{PC} 1$ function, that inadequate effective functional PC1 underlies cyst formation in PCLD, and that PC1 dosage is the rate-limiting determinant of occurrence and severity for both PCLD and ADPKD $(7,23)$.

Mutations in PRKCSH and SEC63 explain approximately $35 \%$ of human PCLD. We analyzed 102 probands in whom we did not find mutations in either of these genes by whole exome sequencing to identify rare heterozygous loss-of-function mutations as candidate genes for PCLD. No predominant gene emerged, suggesting substantial genetic heterogeneity in the remaining PCLD patient group. In a minority of patients, we did find enrichment of heterozygous carrier mutations in PKHD1, the causative gene for autosomal recessive polycystic kidney disease (ARPKD), and heterozygous inactivating mutations in $A L G 8, G A N A B$, and $S E C 61 B$, which all encode ER proteins involved in the same pathway as GII $\beta$ and SEC63. We used functional bioassays of PC1 biogenesis and trafficking to show that inactivation of these ER-associated PCLD candidate genes caused defective maturation and trafficking of PC1 whereas inactivation of PKHD1 had no such effect. Our findings extend the genetic determinants of PCLD to 4 more genes, and with that we have causative mutations in an additional $15 \%$ of our cohort. The findings define essential steps in the biogenesis of mature PC1 protein and extend the hypothesis that PCLD occurs due to reduced effective PC1 function. By extension, the finding that PKHD1 carriers can present with PCLD provides evidence in humans for the hypothesis that the function of the PKHD1 gene product fibrocystin may intersect with polycystin signaling in vivo $(7,23,24)$.

\section{Results}

Description of cohorts. We studied a total cohort of 159 unrelated individuals with clinically defined PCLD in which each proband had at least 10 liver cysts and did not fulfill the diagnostic criteria for ADPKD (4). Twenty-eight of the 159 individuals had previously defined mutations in either of 2 known genes, $P R K C S H$ and SEC63 (Supplemental Table 1; supplemental material available online with this article; https://doi.org/10.1172/JCI90129DS1). The remaining 131 individuals underwent whole exome sequencing, and an additional 25 individuals with loss-of-function mutations in PRKCSH or SEC63 were identified (Supplemental Table 1). Four other probands had non-frameshifting deletions or non-synonymous substitution variants of uncertain pathogenicity in SEC63 or PRKCSH (Supplemental Table 2). After excluding the 53 probands with PCLD due to PRKCSH or SEC63 and the 4 individuals with the indeterminate variants, we arrived at a discovery cohort of 102 unrelated individuals for finding additional PCLD genes. We compared characteristics of this discovery cohort and the PRKCSH/SEC63 group. Affected family members were available for 14 of 57 (25\%) of the PRKCSH/SEC63 probands and for 15 of 102 (15\%) of the unknown cases. In keeping with the increased clinical severity of PCLD in female patients, which likely leads to enhanced ascertainment, 31 of 41 (76\%) of the PRKCSH/SEC63 group and 74 of 102 (73\%) of the discovery cohort were female. Principal component analysis (PCA) comparing all cases that had whole exome sequencing ( $n=131)$ with individuals of defined ancestry from HapMap345 was used to define the ethnic background of PCLD probands in both the PRKCSH/SEC63 and discovery cohorts (Supplemental Table 3). Overall, the discovery and PRKCSH/SEC63 PCLD groups were largely comparable.

Analysis of the discovery cohort. We searched for novel PCLD genes in the discovery cohort by whole exome sequence analysis. We confirmed the unrelated status, defined as identity by descent $<0.125$, of each proband in this group using pairwise comparison of exome data. We prospectively specified as candidates for PCLD all genes with loss-of-function variants (i.e., nonsense, splice site, or frameshift) that had a minor allele frequency (MAF) less than $1 \times 10^{-3}$ in the Exome Aggregation Consortium (ExAC) database. The MAF threshold was chosen conservatively because of uncertainty about the actual prevalence of radiographically defined PCLD. Autopsy studies have suggested prevalence ranging from $1: 400$ to $1: 2,000$, with the largest study showing 1:1,300 (25-27). We found 1,266 sequence variants meeting these criteria in the 102 probands. None of these variants occurred in homozygous or compound heterozygous states, suggesting that PCLD in this cohort is largely a heterozygous trait.

We conducted 2 analyses to evaluate the significance of the per-gene burden of these rare loss-of-function variants. First, a binomial test was used to compare the expected and observed number of variants in each gene. The expected number of lossof-function variants meeting our criteria was determined by taking the fractional length of a gene relative to the entire exome (i.e., the coding length of the transcript divided by the number of bases in the total exome) and multiplying this by 1,266 (the total number of variants). This represents the expected occurrence of sporadic mutations of this type in each gene without considering the influences of "hot spots," pressures of selection, or precedents of ethnic background. A quantile-quantile (Q-Q) plot comparing the distribution of observed and expected probabilities for all genes containing loss-of-function variants in our discovery cohort shows genome-wide significant divergence from the expected for only 2 genes, PKHD1 and ALG8, which encodes a glucosyltransferase (Figure $1 \mathrm{~A}$ and Table 1). Genome-wide significance threshold was taken as $P$ less than $2.4 \times 10^{-6}$ after accounting for examination of 18,715 genes. The expected number of loss-of-function variants in PKHD1 among the 1,266 total variants was 0.48 , but the observed number was $9(P=2.31 \times$ $10^{-9} ; 19$-fold enrichment; Table 1$)$. The expected number of lossof-function variants in $A L G 8$ was 0.06 , and the observed number was $5\left(P=7.18 \times 10^{-9} ; 81\right.$-fold enrichment; Table 1$)$. 

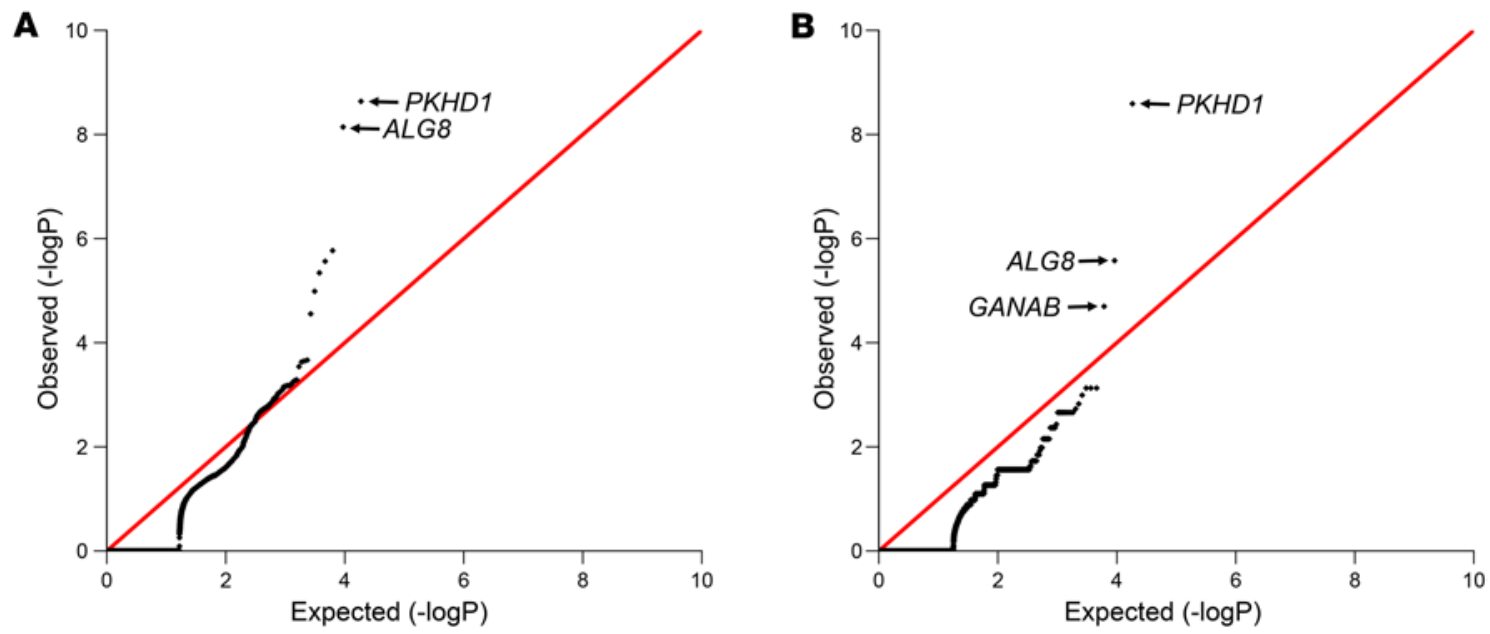

Figure 1. Quantile-quantile plots of observed $\boldsymbol{P}$ values versus an expected distribution of $\boldsymbol{P}$ values. (A) Observed $P$ values calculated using a binomial test by comparison of the observed burden of rare $\left(\mathrm{MAF}<1 \times 10^{-3}\right)$ loss-of-function variants in PCLD cases with the "expected" burden based on the gene transcript length $(n=102)$. (B) Observed burden in the European subset of discovery cohort $(n=92)$ compared with European controls $(n=3,274)$ using Fisher's exact test.

We next performed a case-versus-control analysis of gene burden for the loss-of-function variants in the European subset of our discovery cohort $(n=92)$ versus an available cohort of 3,274 European normal controls sequenced and analyzed by the Yale Center for Mendelian Genomics (28-30). PCA analysis confirmed clustering of the 92 cases and 3,274 controls over European standards (Supplemental Figure 1). The exome coverage metrics met accepted standards, and there was no significant difference in these metrics between cases and controls (Supplemental Tables 4 and 5). A Q-Q plot comparing the observed distribution of Fisher's exact statistics for the frequency of loss-of-function variants in cases versus controls shows genome-wide significance for heterozygous loss-of-function variants only in PKHD1 $\left(P=1.55 \times 10^{-9}\right)$ (Figure 1B and Table 1). The gene with the next lowest $P$ value, $A L G 8(P=2.65$ $\left.\times 10^{-6}\right)$, just missed the threshold for genome-wide significance (Figure 1B and Table 1). Only 4 of 5 ALG8 loss-of-function variants occurred in European cases, yielding 4 of 184 European case alleles compared with 1 of 6,548 European control alleles (Tables 1 and 2). The remaining ALG8 loss-of-function allele occurred in an African American individual. Analysis of a similarly obtained but smaller African American control group (392 individuals, 784 alleles) found no loss-of-function $A L G 8$ alleles. In aggregate, both analyses support PKHD1 and $A L G 8$ as candidate genes for PCLD.

We noted that the next-highest-scoring gene in the caseversus-control analysis was GANAB $\left(P=2.01 \times 10^{-5}\right)$ (Figure 1 and Tables 1 and 2). GANAB encodes the $\alpha$ subunit of GII (GII $\alpha$ ), which is the catalytic subunit that works with the previously defined PCLD gene product GII $\beta$ (encoded by PRKCSH), to form the func-

Table 1. Enrichment of rare heterozygous loss-of-function variants in PKHD1, ALG8, GANAB, and SEC61B in PCLD

\begin{tabular}{|c|c|c|c|c|c|c|c|c|}
\hline \multirow[b]{2}{*}{ Gene } & \multirow[b]{2}{*}{ Protein name } & \multicolumn{4}{|c|}{ Binomial analysis ${ }^{B}$} & \multicolumn{3}{|c|}{ European case vs. control ${ }^{\mathrm{C}}$} \\
\hline & & Observed ( $n$ ) & Expected & Fold change & $P$ value & Cases (n) & Controls (n) & $P$ value \\
\hline \multirow[t]{2}{*}{ PKHD1 } & Fibrocystin/Polyductin & 9 & 0.48 & 18.8 & $2.31 \times 10^{-9}$ & 9 & 12 & $1.55 \times 10^{-9}$ \\
\hline & & & & & & $10^{0}$ & $16^{D}$ & $6.63 \times 10^{-10}$ \\
\hline ALG8 & $\alpha-1,3-G l u c o s y l t r a n s f e r a s e$ & 5 & 0.06 & 80.7 & $7.18 \times 10^{-9}$ & $4^{\mathrm{E}}$ & 1 & $2.65 \times 10^{-6}$ \\
\hline GANAB & Glucosidase Il $\alpha$ & 3 & 0.11 & 26.4 & $2.25 \times 10^{-4}$ & 3 & 0 & $2.01 \times 10^{-5}$ \\
\hline \multirow[t]{2}{*}{ SEC61B } & SEC61 $\beta$ & 1 & 0.01 & 87.2 & $1.14 \times 10^{-2}$ & 1 & 0 & $2.73 \times 10^{-2}$ \\
\hline & & & & & & $2^{F}$ & 0 & $7.43 \times 10^{-4}$ \\
\hline
\end{tabular}

${ }^{A}$ Only loss-of-function variants (frameshift, nonsense, splice site) with MAF less than $1 \times 10^{-3}$ are included, except as noted. All variants were verified by direct Sanger sequencing of amplicons. No individual among either cases or controls had homozygous loss-of-function variants in these genes. ${ }^{\mathrm{B} T o t a l}$ discovery cohort, $n=102$. $P$ value is determined by the binomial test. 'European discovery cohort cases, $n=92$ ( $n=184$ alleles). European controls, $n=3,274$ ( $n=6,548$ alleles). $P$ value is calculated using Fisher's exact test comparing allele counts. ${ }^{~ I n c l u d e s ~ t h e ~ p . T 36 M ~ m u t a t i o n, ~ w h i c h ~ i s ~ a ~ f o u n d e r ~ m u t a t i o n ~ i n ~}$ Europeans and is the most common pathogenic variant for ARPKD (17\% of mutant alleles) (41). MAF for this variant is $8.55 \times 10^{-4}$ in non-Finnish Europeans in ExAC. It is present in 1 case in the discovery cohort and 4 members of the control population. Since this variant does not strictly meet our definition for loss-of-function variants, we provide $P$ values for PKHD1 both excluding and including the p.T36M variant. EOnly 4 of 5 cases in our discovery cohort with loss-of-function variants in ALG8 are European. The fifth case is African American. FIncludes a non-synonymous substitution variant, p.M1V, which is predicted to cause loss of translation since there is no alternative in-frame initiation codon. This variant is not present in 3,274 European controls. ExAC has 1 allele with a different sequence change that also results in loss of the start codon (p.M1L, 1 of 121,342 alleles). 
Table 2. Heterozygous mutations in ALG8, GANAB, SEC61B, and PKHD1 that result in PCLD

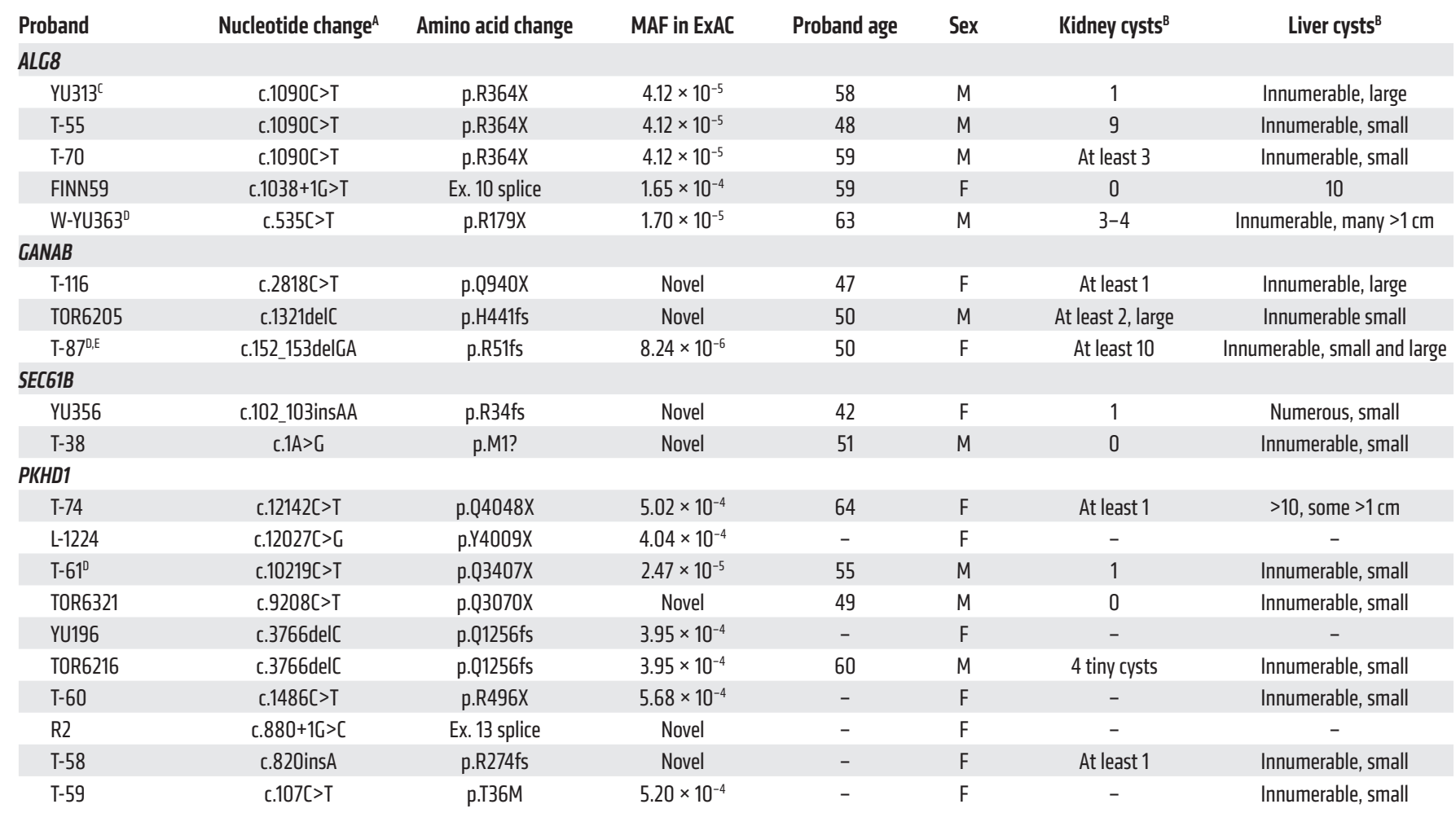

${ }^{A}$ All variants were found by whole exome sequencing and confirmed with Sanger sequencing. ${ }^{B}$ Cyst counts are made from available reports or imaging. ${ }^{C}$ African American. All other probands were European. ${ }^{D}$ Affected first-degree relative (sibling or child) segregation data are available only for these 3 patients. Affected relatives also have the respective variant. ${ }^{E}$ As this article was being prepared, the GANAB mutation in this patient's sister, but not in this patient, was reported by Porath et al. (48).

tional GII holoenzyme in the ER lumen. Two of the three GANAB variants in our cohort are novel, and the third is exceedingly rare, with MAF less than $1 \times 10^{-5}$ (Table 2 ). In the analysis based on the binomial distribution, GANAB had $P=2.25 \times 10^{-4}$ with a predicted burden of 0.11 and an enrichment of 26 -fold in the 3 observed cases (Table 1). GANAB is a highly loss-intolerant gene, with a probability of loss-of-function intolerance score (pLI) of 1 in ExAC, whereas PKHD1 and ALG8 are not loss intolerant, consistent with genes associated with a recessive human disease (31). To evaluate $G A N A B$ further, we performed a follow-up analysis using the binomial test including only genes with very rare loss-of-function variants $\left(\mathrm{MAF}<5 \times 10^{-5}\right)$ and $\mathrm{pLI}$ greater than 0.9 in our PCLD cohort. The observed distribution for all genes conforms closely to the expected with the exception of GANAB, which diverged significantly. The expected number of loss-of-function alleles for GANAB was 0.02 , while the observed number was $3(P=1.46 \times$ $10^{-6} ; 143$-fold enrichment).

Finally, we noted a novel loss-of-function mutation in SEC61B, encoding a component of the SEC61 ER membrane proteinconducting channel that associates with SEC63 (32). There are no loss-of-function mutations for SEC61B in ExAC. We found a second patient with a novel, likely loss-of-function missense variant in SEC61B that mutated the initiation codon, leaving no apparent in-frame alternative start site for translation (Table 2). While it did not reach statistical significance on a genome-wide level, SEC61B showed 87-fold change in the binomial analysis, and, if both variants are considered in the case-versus-control analysis, SEC61B had $P=7.43 \times 10^{-4}$ (Tables 1 and 2).

Further independent validation of these analyses was done using the European non-Finnish and Finnish combined subset of the population in the ExAC database. Gene-by-gene comparison showed enrichment of mutations for each of our candidate genes in PCLD cases: PKHD1, $P=2.34 \times 10^{-8}$; ALG8, $P=2.59$ $\times 10^{-5}$; GANAB, $P=5.52 \times 10^{-6}$; SEC61B, $P=6.46 \times 10^{-6} . A L G 8$, $G A N A B$, and $S E C 61 B$ all function in the common ER biogenesis pathway defined by SEC63 and PRKCSH. The increased occurrence of loss-of-function mutations and convergence on a single pathway known to be causative in PCLD prompted us to investigate these 3 genes as functional candidates for causing PCLD. In addition, the genome-wide significant enrichment for PKHD1 in our PCLD cohort prompted us to further explore the association of PKHD1 with PCLD.

Functional validation of candidates. We had previously shown that somatic homozygous inactivation of Prkcsh and Sec63 in vivo results in polycystic kidney and liver disease due to defective biogenesis and trafficking of $\operatorname{PC} 1(7,23,33)$. This mechanistic understanding indicated that we can validate new candidate genes for PCLD by determining the effects of inactivation of each gene on alterations in its posttranslational maturation of PC1. Full-length PC1 (PC1-FL) undergoes autoproteolytic cleavage at the GPCR 
A

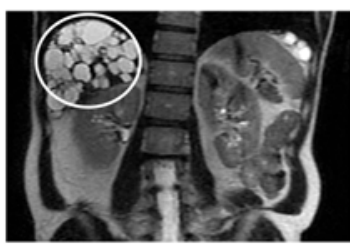

B

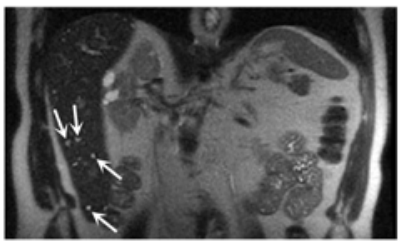

E

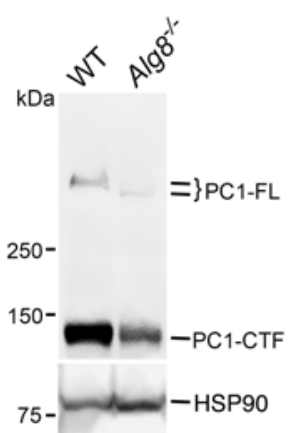

$\mathbf{F}$

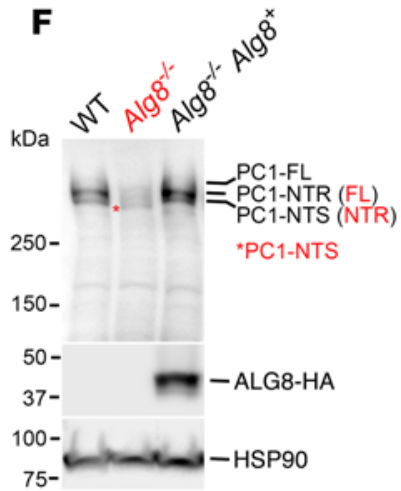

C

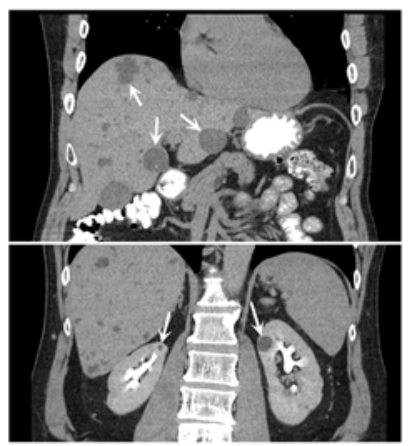

G
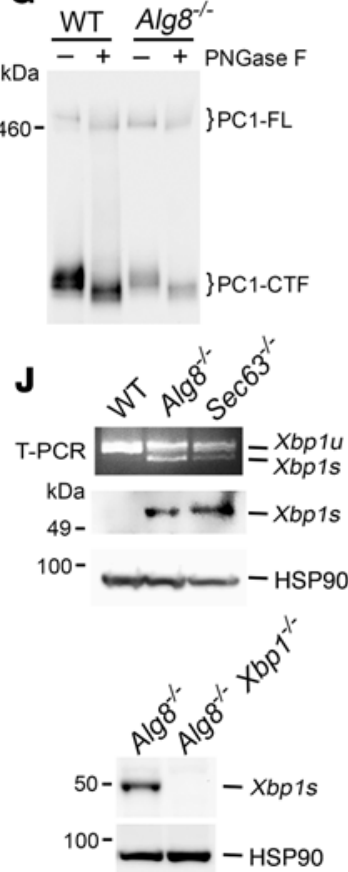

D

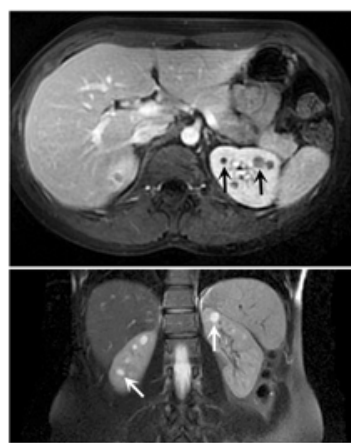

H

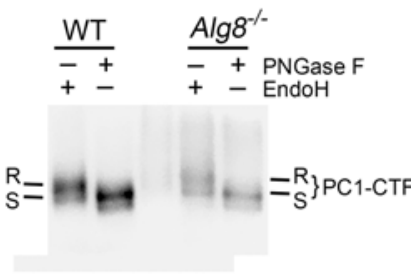

$\mathbf{K}$

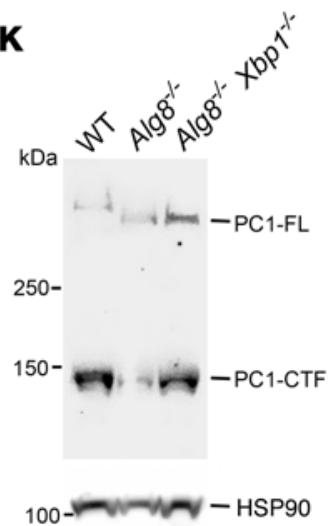

Figure 2. Mutations in ALG8 cause abnormal biogenesis of PC1. (A) YU313 has innumerable liver cysts larger than $1 \mathrm{~cm}$ (circle). (B) T-70 has innumerable small liver cysts (arrows). (C) W-YU363 has innumerable liver cysts, many larger than $1 \mathrm{~cm}$ (arrows, top panel), and 3-4 kidney cysts (arrows, bottom panel), while his 19-year-old daughter, YU364 (D), has the same ALG8 mutation but 8 kidney cysts (arrows, top and bottom panels) and no liver cysts. (E and F) Immunoblots of cell lysates with anti-HA (E) and anti-LRR PC1 N-terminal antibody (7e12) (F) show decreased PC1-CTF, PC1-FL, PC1-NTR, and PC1-NTS in Alg $8^{-/-}$cells. Re-expression of $A / g 8^{+}$in $A / g 8^{-/-}$cells rescues PC1 expression (F). The labels in red mark the migration of the respective PC1 fragments in the Alg $8^{-1-}$ cell lysate. (G and $\mathbf{H}$ ) Anti-HA immunoprecipitation of PC1-FL and PC1-CTF was treated with PNGase F or EndoH. (G) Altered migration of PC1-FL is due to hypoglycosylation in Alg8 ${ }^{-/-}$cells. PNGase $\mathrm{F}$ treatment shows equivalent deglycosylated PC1-FL migration. (H) The relative proportion of EndoHresistant/EndoH-sensitive PC1-CTF is reduced in Alg $8^{-/-}$cells. In $\mathbf{G}$ and $\mathbf{H}$, the 2 PC1-CTF bands in the PNGase F-treated lanes result from alternatively spliced forms present in rodents (66). (I) Immunofluorescence shows absence of detectable PC1 (anti-HA) in cilia of Alg $8^{-1-}$ cells. (J) Alg $8^{-/-}$cells activate the IRE1 $\alpha /$ XBP1 branch of UPR, demonstrated by presence of XBP1s by reverse transcription PCR (RT-PCR; top) and immunoblotting (bottom). (K) Anti-HA immunoblot shows that inactivation of both Alg8 and Xbp1 does not change PC1 hypoglycosylation or GPS cleavage compared with Alg8 knockout alone. HSP9O serves as loading control for cell lysates.

proteolysis site (GPS) into an approximately 3,000-amino acid extracellular N-terminal fragment (PC1-NTF) and an approximately 1,300-amino acid intramembranous C-terminal fragment (PC1-CTF) $(34,35)$. PC1-NTF migrates as 2 protein species - the cell surface-expressed endoglycosidase $\mathrm{H}$-resistant (EndoHresistant) PC1-NTR and the intracellular EndoH-sensitive PC1NTS $(36,37)$. For the current study, we examined PC1 steady-state protein expression level and trafficking using a kidney epithelial cell line generated from mouse models containing 3 copies of a $\mathrm{BAC}$ transgene expressing $P k d 1$ with $\mathrm{N}$-terminal FLAG and C-ter- minal HA epitope tags $\left(P k d 1^{\mathrm{F} / H}-B A C\right)(7,33,35)$. We used CRISPR/ Cas9 genome editing methods to inactivate each candidate gene in the cells expressing $P k d 1^{\mathrm{FH}-B A C}$ and investigated the impact on the PC1 protein.

ALG8. ALG8 (asparagine-linked glycosylation 8) encodes $\alpha$-1,3-glucosyltransferase, an ER integral membrane protein that functions to add the second of 3 glucoses to the assembling lipidlinked oligosaccharide precursor for $\mathrm{N}$-linked glycosylation before it is transferred to the nascent peptide (38). ALG8 intersects with the protein biogenesis pathway between the sites of action of 
SEC63 and GII $\beta$. Patients with ALG8 mutations showed variation in severity of PCLD and frequent presence of kidney cysts (Figure 2, A-D, and Table 2). Four probands, YU313, T-70, T-55, and $\mathrm{W}-\mathrm{YU} 363$, are male and have nonsense codon mutations in $A L G 8$. Three of these probands, 2 European and 1 African American, shared the same mutation, p.R364X (Table 2). Identity-by-descent analysis found that the largest possible shared haplotype block among any 2 of these individuals is approximately $87 \mathrm{~kb}$. This is too small to be indicative of a cryptic relationship between any of the probands and instead suggests that the mutation in each arose independently. FINN59 is a Finnish woman with a mild presentation carrying a splice mutation found with a frequency of 1.66 $\times 10^{-3}$ in the Finnish population in ExAC. YU313 has symptomatic PCLD with many cysts larger than $1 \mathrm{~cm}$ (Figure 2A), while T-70 (Figure 2B) and T-55 (not shown) have numerous small liver cysts. W-YU363 has innumerable liver cysts of varied sizes (Figure 2C), but his daughter has 8 kidney cysts without any liver cysts (Figure 2D). She shares the $A L G 8$ mutation with her father, and complete clinical testing for PKD1 and PKD2 mutations was negative; her mother has no kidney or liver cysts.

We used CRISPR/Cas9 to inactivate both Alg8 alleles in our mouse epithelial cell line expressing $P k d 1^{F / H}-B A C$. Sanger sequencing confirmed compound heterozygous frameshifting loss-of-function mutations in Alg8-null cells ( Alg $^{-/}$; Supplemental Table 6). Alg8 ${ }^{-/}$cells showed reduced levels of PC1FL and PC1-CTF detected by the anti-HA C-terminal epitope tag (Figure 2E). PC1-NTR and PC1-NTS detected by the anti$\mathrm{N}$-terminal native protein antibody were also reduced (Figure $2 \mathrm{~F})$. The 2 antibodies are detecting different pools of PC1 in these cells. The anti-HA antibody is detecting PC1 expressed from the $P k d 1^{F / H}-B A C$ transgene, whereas the N-terminal anti-LRR native $\mathrm{PC} 1$ antibody (7e12) is primarily detecting $P k d 1$ expressed from the endogenous gene (Supplemental Figure 2). The presence of the FLAG epitope in the transgenically expressed PC1 appears to impair recognition of the native epitope. There was no difference in $P k d 1$ transcript expression between WT and $A l g 8^{-/-}$cells (data not shown), indicating that differences in steady-state protein level are a post-transcriptional effect. Examination of integral membrane proteins from other membrane compartments, i.e., PC2 (ER, cilia), $\mathrm{Na}^{+} / \mathrm{K}^{+}$-ATPase $\alpha_{1}$ subunit (plasma membrane), and calnexin (ER), did not show significant quantitative reductions, indicating that not all membrane proteins are similarly affected by inactivation of Alg8 (Supplemental Figure 3A). Since CRISPR/Cas9 can produce off-target effects, we showed the specific dependence of these findings on ALG8 by rescuing the protein expression level of PC1 with re-expression of Alg8 cDNA in Alg8 ${ }^{--}$cells (Figure $2 \mathrm{~F}$ ).

The electrophoretic migration of PC1-FL was notably faster in Alg8 $8^{-1}$ cells (Figure 2, E-G and $\mathrm{K}$ ), suggesting that PC1 may have a defect in glycosylation in addition to reduced protein quantity in the absence of ALG8. Immunoprecipitation of PC1FL and PC1-CTF followed by treatment with peptide- $N$-glycosidase $\mathrm{F}$ (PNGase F) resulted in the same electrophoretic migration for the protein backbones irrespective of Alg8 genotype, confirming that differences in glycosylation account for the genotypedependent altered gel mobility of PC1-FL in cell lysates (Figure $2 \mathrm{G})$. The data show that effective reduction in PC1 function in the absence of ALG8 is likely to result from the combined effect of the quantitative decrease in steady-state levels of PC1 and the structural abnormality of PC1 resulting from defective posttranslational modification. N-glycosylated proteins typically acquire resistance to EndoH as they transit past the middle Golgi on the way to the cell surface. The relative proportion of EndoH-resistant PC1-CTF to EndoH-sensitive PC1-CTF is markedly reduced in Alg8 $8^{-/}$cells (Figure $2 \mathrm{H}$ ), providing biochemical evidence that PC1 trafficking to the cell surface, and by extension to cilia, is markedly impaired following inactivation of Alg8. In keeping with this, immunofluorescent cell staining with anti-ARL13B to mark cilia and anti-HA to detect PC1 shows absent or markedly reduced visualization of PC1 in cilia in Alg $8^{-/-}$cells compared with cells expressing Alg8 normally (Figure 2I).

We had previously shown that inactivation of Sec63, but not of Prkcsh, results in activation of the IRE1 $\alpha / \mathrm{XBP} 1$ branch of the unfolded protein response (UPR) (33). Activation of this branch of UPR results in formation of XBP1s, the active spliced form of $\mathrm{XBP} 1$, which acts as a transcription factor for an array of chaperone proteins (39). Alg8 $8^{-/}$cells also have activation of the IRE1 $\alpha$ / $\mathrm{XBP1}$ branch of UPR as evidenced by reverse transcription PCR and immunoblot assays showing the presence of XBP1s (Figure 2J). In the absence of SEC63, active XBP1s is required to enable autoproteolytic cleavage of PC1 at the GPS (33). To determine whether XBP1s has a similar role in $A l g 8^{-/-}$cells, we inactivated $\mathrm{XBP1}$ in $\mathrm{Alg} 8^{-/-}$cells to make double-knockout cells $\left(\mathrm{Alg}^{-/-} \mathrm{Xbp1}^{-/-}\right.$; Figure $2 \mathrm{~K}) . \mathrm{Alg}^{-/-} \mathrm{Xbp1}^{-/-}$cells showed normal cleavage of PC1 (Figure 2K), indicating that while the IRE1 $\alpha / \mathrm{XBP} 1$ branch of UPR is activated following loss of ALG8, it is not required for PC1 cleavage. In aggregate, the relative enrichment of rare loss-of-function variants of $A L G 8$ in PCLD patients coupled with functional evidence that inactivation of ALG8 affects PC1 biogenesis and trafficking supports the conclusion that $A L G 8$ mutations are a genetic cause of PCLD.

GANAB. Three PCLD patients, but none in the control population, had loss-of-function mutations in GANAB encoding GII $\alpha$ (Tables 1 and 2). The severity of the liver phenotype in GANAB patients varied from numerous large liver cysts with hepatomegaly (Figure 3A) to innumerable small cysts (Figure 3B). All 3 patients had at least 1 kidney cyst, but only 1 had more than 10 (Figure 3, A and B, and Table 2). To assess the functional consequences of loss of Ganab, we inactivated the gene in the cells expressing $P k d 1^{F / H}-B A C$ using CRISPR/Cas9. We confirmed inactivation of Ganab by Sanger sequencing that showed homozygous deletion of $281 \mathrm{bp}$ beginning in exon 12 and extending into intron 13 (Supplemental Table 6) and by anti-GANAB immunoblot of cell lysates (Figure $3 \mathrm{C}$ ). Ganab ${ }^{-/}$cells showed markedly reduced or absent PC1-NTR (Figure 3C) and decreased steadystate levels of PC1-FL and PC1-CTF (Figure 3D). The $\mathrm{Na}^{+}$/ $\mathrm{K}^{+}$-ATPase $\alpha_{1}$ subunit protein was also decreased in the absence of GII $\alpha$, suggesting effects on at least some other plasma membrane proteins (Supplemental Figure 3, B and C). Functional re-expression of GII $\alpha$ in $\mathrm{Ganab}^{-/}$cells rescued the expression of PC1-NTR, showing that the observed effects on PC1 protein maturation were solely the result of GII $\alpha$ deficiency (Figure 3C).

A reproducible $(n=4)$ feature of $\mathrm{Ganab}^{-/-}$cells was that PC1FL and PC1-NTS migrated more slowly (Figure 3, C and D). We 
A

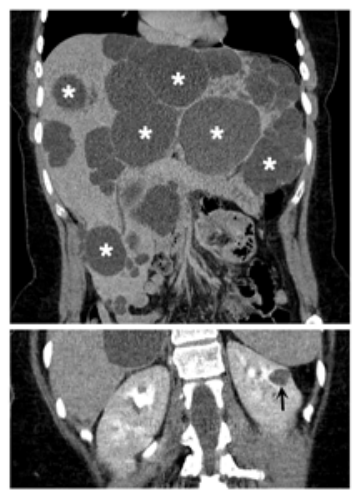

B

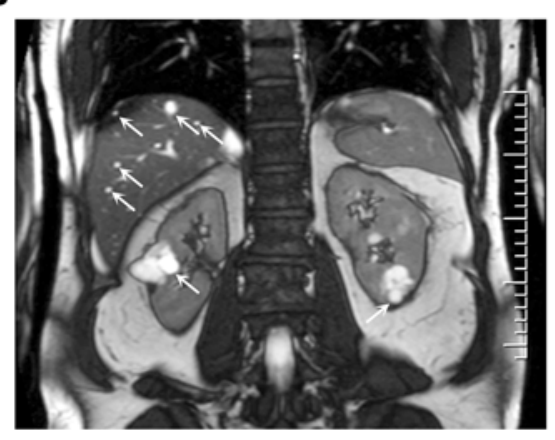

C

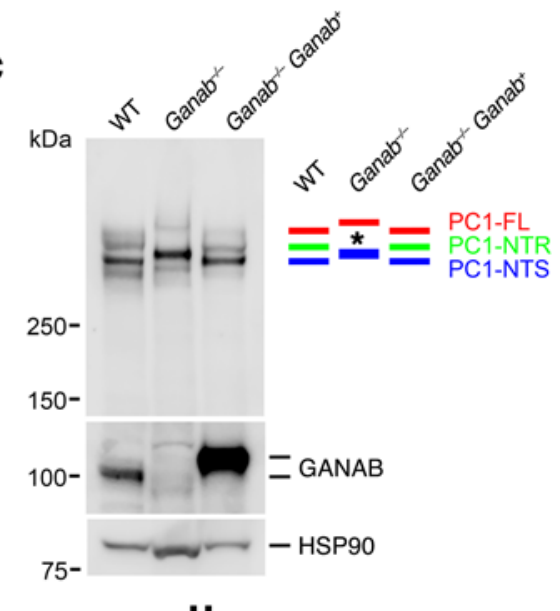

D

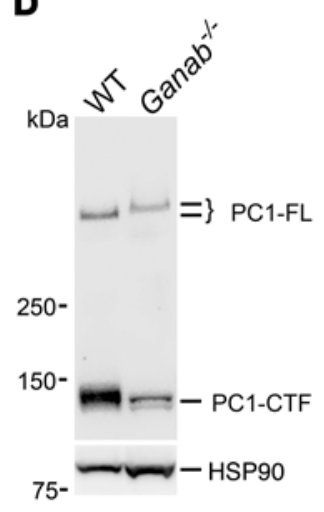

E

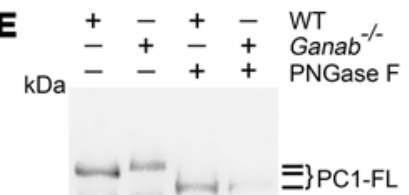

$\mathrm{kDa}$

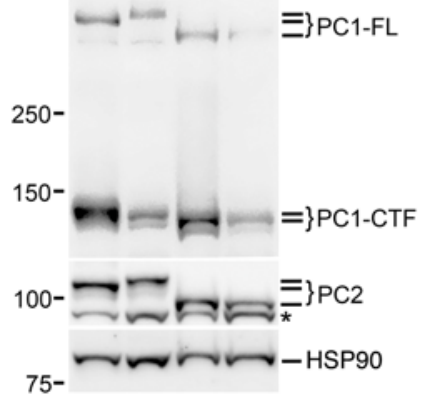

$\mathbf{F}$

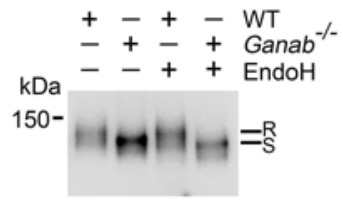

G

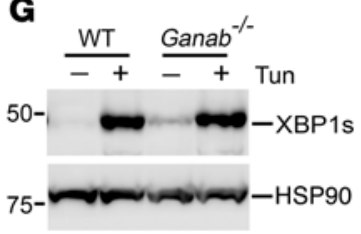

H

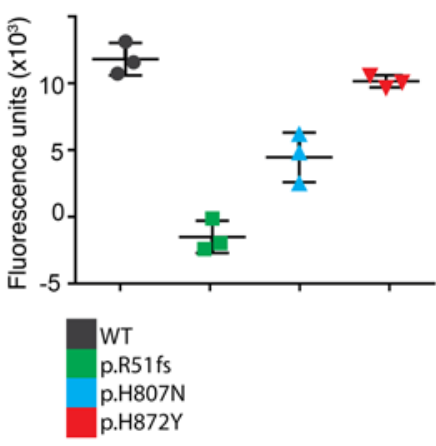

Figure 3. Mutations in GANAB cause PCLD. (A) T-116 has innumerable large liver cysts (asterisks) with resultant hepatomegaly. (B) TOR6205 has innumerable small liver cysts (arrows). Kidney cysts are present in both cases (arrows). (C and D) Reduced protein expression and altered migration of PC1 fragments in Ganab ${ }^{-/-}$cells. Immunoblot of cell lysates with anti-PC1-LRR N-terminal antibody (C, top panel) and anti-GANAB (C, Gll $\alpha$; middle panel) demonstrates absence of PC1-NTR (asterisk) in Ganab ${ }^{-1-}$ cells. Anti-HA immunoblot (D) shows significant decrease in PC1-CTF. PC1-FL (C and D) and PC1-NTS (C) have slower migration in Canab ${ }^{-/}$cells. The PC1 expression level and altered gel-migration pattern are rescued by re-expression of Canab (C, $\mathrm{Canab}^{-1-}$;Ganab ${ }^{+}$). The re-expressed Gll $\alpha$ is larger than the native protein because of the inclusion of an epitope tag and an alternatively spliced exon 6 that is absent from the native protein in the mouse cell line. (E) PC2 and PC1-FL show higher molecular mass in Ganab ${ }^{-/-}$cells that resolves to the same gel migration in WT cells following PNGase F treatment. ${ }^{*}$ Nonspecific band. (F) Ganab ${ }^{-1-}$ cells only have EndoH-sensitive (S) and lack EndoH-resistant (R) PC1-CTF. PC1-CTF shows 2 bands due to alternative spice forms in rodents. (C) IRE1 $\alpha /$ XBP1 branch of UPR is activated in Canab ${ }^{-/-}$cells as evidenced by increased XBP1s. Tunicamycin (Tun) treatment $(2.5 \mu \mathrm{g} / \mathrm{ml}$ for 6 hours) serves as positive control for UPR activation. (H) Functional assay of GANAB missense variants. Fluorescence output from the glucosidase II fluorogenic substrate 4-methylumbelliferyl $\alpha$-D-glucopyranoside (4-MUG) from HEK 293T cells cotransfected with GII $\beta$ and the respective variants of GIl $\alpha$. Background fluorescence from untransfected cell lysates is subtracted. HSP9O serves as loading control for cell lysates.

had previously shown that there is aberrant glucose trimming with GII $\beta$ mutations (40), so the slower electrophoretic migration of PC1 is likely to be the result of a similar defect following loss of GII $\alpha$. In keeping with this, removal of all N-glycans by PNGase $\mathrm{F}$ resulted in elimination of the differential migration of PC1 (Figure 3E). Although there was no striking difference in PC2 levels in $\mathrm{Ganab}^{-/}$cells, PC2 also showed an aberrant migration pattern that disappeared after PNGase F treatment (Figure 3E). While PC1 defects are likely to be rate-limiting in the polycystic phenotype $(7,23)$, aberrant glycosylation of PC2 may contribute to functional deficiency of the polycystin complex. We further examined the genotype-dependent glycosylation and trafficking properties of PC1-CTF by immunoprecipitation using anti-HA, followed by EndoH treatment. PC1-CTF had markedly reduced or absent EndoH-resistant forms in $\mathrm{Ganab}^{-/-}$cells (Figure 3F), further indicating that beyond decreased absolute PC1 protein levels, the residual protein is abnormally processed and has markedly impaired trafficking past the Golgi. While there is no activation of the IRE1 $\alpha / \mathrm{XBP} 1$ branch of UPR by inactivation of GII $\beta$ (33), we found that loss of GII $\alpha$ resulted in increased formation of the active XBP1s, suggesting a somewhat more severe cellular stress with inactivation of the catalytic subunit of GII (Figure 3G).

Two patients in our cohort had rare, potentially deleterious GANAB missense variants p.H807N and p.H872Y (Supplemental Table 7). Each occurred in individuals in whom a causative PCLD gene mutation in $A L G 8$ and $P K H D 1$, respectively, had been identified. We expected that these GANAB variants do not result in complete loss of function and are therefore not causative for PCLD. To test this hypothesis, we assayed the catalytic activity of both GII $\alpha$ variants and compared them with WT GII $\alpha$ and with a known lossof-function mutant we had identified, p.R51fs. HEK 293T cells were cotransfected with GII $\beta$ and each of the 4 GII $\alpha$ variants. Cell 
A

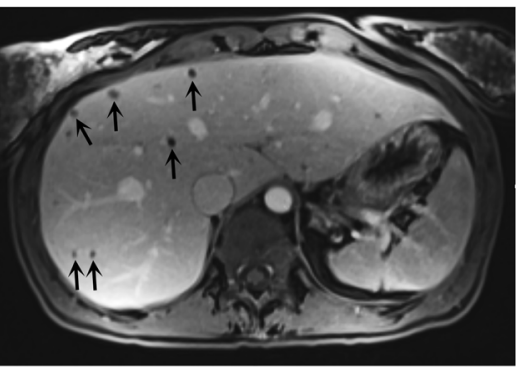

C
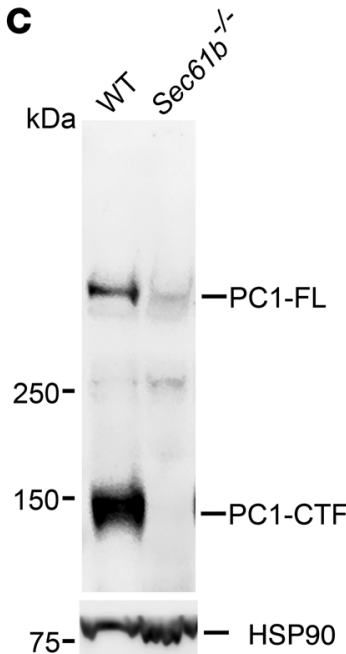

75- - HSP90

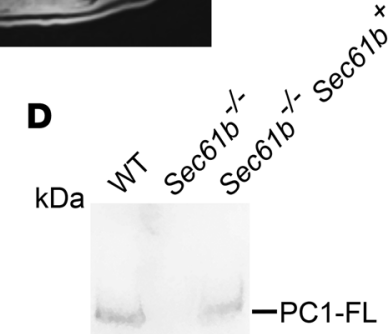

$250-$
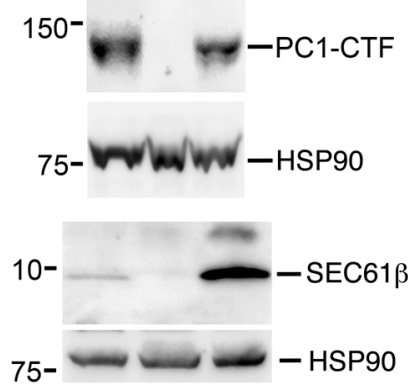

B

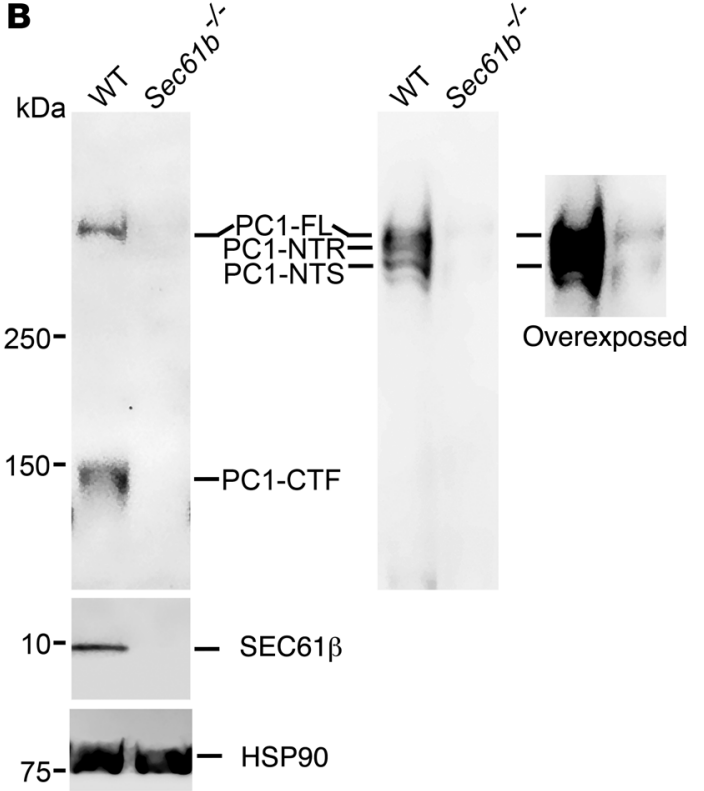

$\mathbf{E}$

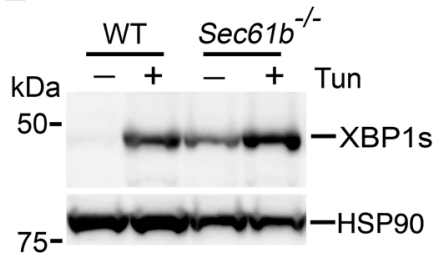

Figure 4. Mutations in Sec61b have profound effects on quantity and maturation of PC1. (A) Liver imaging for YU356 showing numerous small liver cysts (arrows). (B) Immunoblots of cell lysates with C-terminal (anti-HA; left) and N-terminal (anti-LRR, 7e12; right) antibodies show that all PC1 fragments are markedly decreased to almost undetectable levels in Sec61 $b^{-1-}$ cells. PC1-NTR is absent even on long exposure. Immunoblot with anti-SEC61 $\beta$ antibody shows absence of SEC61 $\beta$ protein in Sec61 $6 b^{-/-}$cells. WT, isogenic wild-type control cells. (C) Long-exposure immunoblot of cell lysates with anti-HA showing a minute amount of PC1-FL but complete absence of PC1-CTF. (D) Immunoblots of cell lysates with anti-HA showing complete rescue of PC1 phenotype with stable re-expression of Sec61b $\left(\operatorname{Sec} 61 b^{-/-} ; \operatorname{Sec}_{61 b^{+}}\right)$. (E) Activation of the IRE1 $\alpha /$ XBP1 branch of UPR as evidenced by increased levels of XBP1s in Sec61 $\mathrm{b}^{-/-}$cells. Tunicamycin (Tun) treatment $(2.5 \mu \mathrm{g} / \mathrm{ml}$ for 6 hours) serves as positive control for UPR activation. HSP9O serves as loading control for all immunoblots.

lysates were incubated with the GII fluorogenic substrate 4-methylumbelliferyl $\alpha$-D-glucopyranoside (4-MUG), and fluorescence output above the baseline values obtained using untransfected cell lysates was used to quantify GII enzymatic activity (Figure $3 \mathrm{H}$ and ref. 40). The activity of p.H872Y was similar to WT, while p.H807N showed approximately $50 \%$ reduction in activity (Figure $3 \mathrm{H}$ ). The pathogenic variant p.R51fs showed complete loss of activity. While these missense variants in GII $\alpha$ are not pathogenic in PCLD, it remains possible that heterozygous hypomorphic variants such as p.H807N could have genetic modifier effects on the expressivity of PCLD due to mutations in other causative genes (e.g., PKHD1).

SEC61B. The SEC61 protein translocation pore consists of 3 subunits, $\alpha, \beta$, and $\gamma$. The $\beta$ subunit, encoded by SEC61B, is the least conserved component and the one that is not essential for SEC61 complex function (32). Two patients had heterozygous lossof-function variants in SEC61B (Tables 1 and 2). In both patients, PCLD is characterized by diffuse and numerous small liver cysts (Figure 4A). We inactivated SEC61 $\beta$ in the $P k d 1^{F / H}-B A C$ cells and confirmed complete loss of function by both direct sequencing of cell genomic DNA (Supplemental Table 6) and immunoblotting with native anti-SEC61 $\beta$ antisera (Figure 4B). Inactivation of SEC61 $\beta$ resulted in almost undetectable levels of PC1 when examined by either C-terminal anti-HA epitope antibody or N-terminal native protein antibody (Figure 4B). Very long exposure of the latter immunoblot showed a minute amount of PC1-FL and PC1NTS, but no PC1-NTR was detectable in Sec $61 b^{-/-}$cells (Figure 4B). In some experiments, a long exposure of a C-terminal anti-HA immunoblot could detect minute amounts of PC1-FL, but PC1CTF remained completely undetectable (Figure 4C). As with the other PCLD genes, inactivation of SEC61 $\beta$ had variable effects on other integral membrane proteins $-\mathrm{Na}^{+} / \mathrm{K}^{+}$-ATPase $\alpha_{1}$ subunit was markedly reduced, PC2 was minimally affected, and calnexin was unchanged (Supplemental Figure 3, C-E). Re-expression of SEC61 $\beta$ in Sec $61 b^{-/-}$cells reconstituted PC1 levels including PC1CTF, confirming the specificity of this effect (Figure 4D). As an additional indication that SEC61 $\beta$ inactivation has severe effects 
A

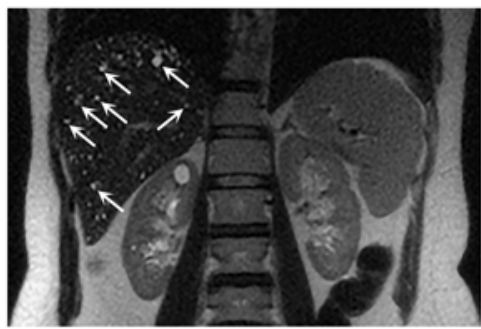

C

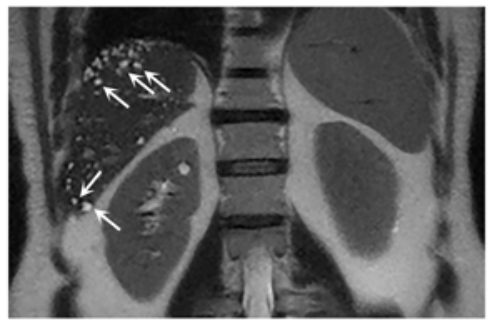

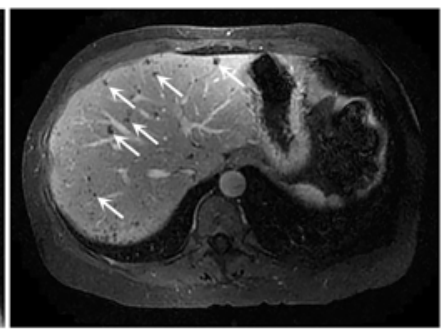

D

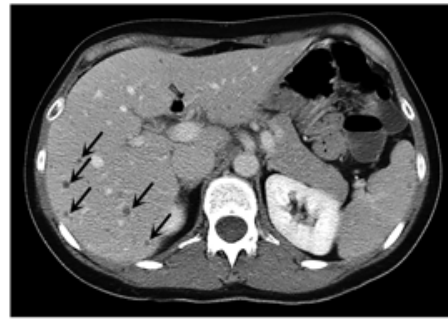

B

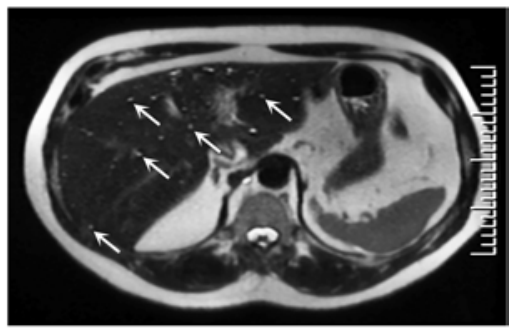

E

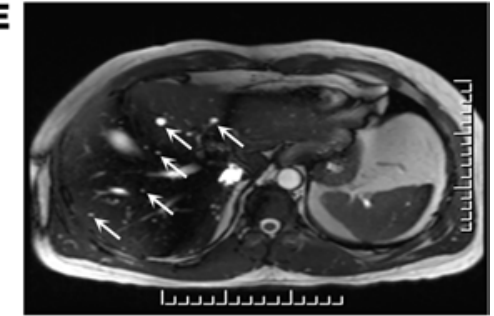

$\mathbf{F}$

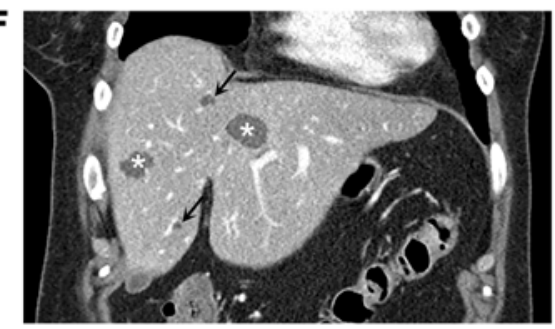

H

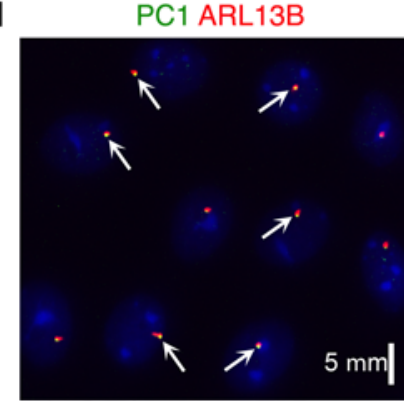

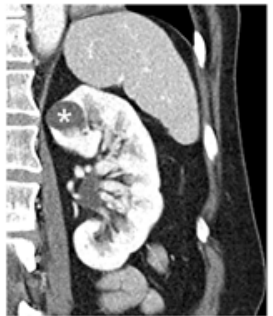

PC1

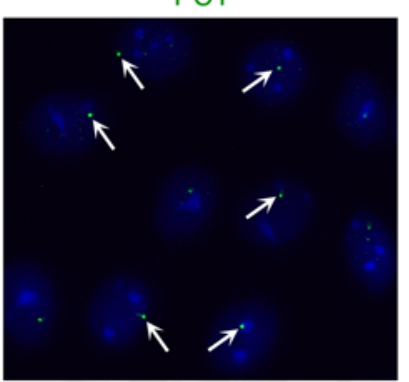

G

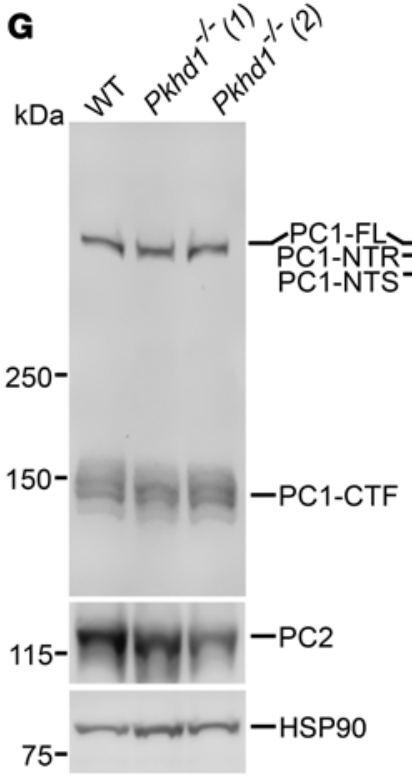

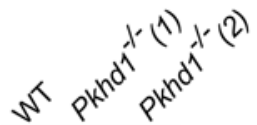

Figure 5. Adult carrier parents for ARPKD can develop PCLD. (A-F) Liver and kidney imaging for cases with heterozygous PKHD1 loss-of-function mutations. T-58 (A), TOR6216 (B), T-61 (C), T-59 (D), and TOR6321 (E) have innumerable small liver cysts (arrows). (F) T-74 has more than 10 liver cysts (arrows and asterisks), some greater than $1 \mathrm{~cm}$ in diameter (asterisks), and at least 1 kidney cyst larger than $1 \mathrm{~cm}$ (asterisk). (C) Immunoblots of cell lysates with the PC1 C-terminal epitope (anti-HA, left) and PC1 N-terminal anti-LRR antibody (7e12, right) using WT cells and 2 independent $P$ khd1 $7^{-/-}$cell lines showing no significant difference in any PC1 fragment and no change in PC2 quantity. HSP90 serves as loading control. (H) Pkhd1/- cells show positive PC1 by anti-

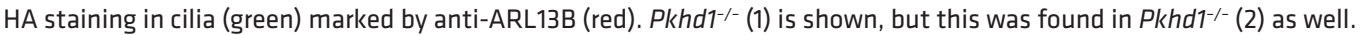

on cellular protein biogenesis, $\operatorname{Sec} 61 b^{-/-}$cells showed the strongest relative activation of XBP1s among all of the PCLD gene cellular knockouts (Figure 4E). In aggregate, the data show that loss of SEC61 $\beta$ results in the most severe quantitative reduction of PC1 among the PCLD genes.

PKHD1. Patients were ascertained for this study based solely on imaging criteria defining PCLD. Whole exome analysis of the discovery cohort identified heterozygous carriers for lossof-function variants in PKHD1, the ARPKD gene, in 9 individuals (Tables 1 and 2). A tenth individual had the p.T36M missense variant in PKHD1 (Table 2), which is a known pathogenic founder mutation in Europeans and the most common mutant allele
(17\%) in ARPKD (41). Therefore, a minimum of 10 of 102 PCLD probands in our discovery cohort are explained by mutations in PKHD1. This significant enrichment of heterozygous pathogenic PKHD1 variants in the PCLD cohort relative to the observed occurrence of these mutations in the general population achieved genome-wide significance (Figure 1 and Tables 1 and 2). The clinical presentation of most of these patients showed innumerable small liver cysts (Figure 5, A-E, and Table 2). This is in contrast to the more typical cyst architecture of PCLD in patients with SEC63, PRKCSH, ALG8, or GANAB variants, which often includes multiple large cysts greater than $1 \mathrm{~cm}$ in size. This may indicate dependence of the clinical phenotype of the PCLD presentation 




Figure 6. Schematic of the function of the PCLD genes in the ER protein biogenesis pathway. The PCLD genes are numbered 1-5. Lipid-linked oligosaccharide precursors of $\mathrm{N}$-linked glycans are initially assembled on dolichol on the cytoplasmic aspect of the ER membrane. These are flipped into the ER lumen, where ALG8 (1) catalyzes the addition of the second glucose residue. Nascent polypeptides undergo cotranslational translocation via the SEC61 translocation pore that is composed of $\alpha, \beta(\mathbf{2})$, and $\gamma$ subunits and is associated with SEC62. SEC63 (3) and ERJ1 act in concert with the major ER HSP70 chaperone BiP to facilitate this translocation process. Oligosaccharyl transferase (OST) catalyzes the attachment of the glycan moiety to asparagine residues. Glucosidase I removes the outermost glucose before glucosidase II, composed of GIl $\alpha$ (4) and GII 3 (5) subunits, removes the second glucose. This step is necessary for the nascent peptide to enter the calnexin (CNX)/calreticulin (CRT) protein folding and quality control cycle. Glucosidase II (4, 5) subsequently removes the innermost glucose from the N-linked glycan, allowing for exit from the CNX/CRT cycle. If the protein has attained its properly folded conformation, it proceeds along the secretory pathway. Misfolded proteins are recognized and reglucosylated by UGGT, allowing for more time in the folding environment of the CNX/ CRT cycle. Eventually, proteins that fail to fold properly undergo ER-associated degradation by retrotranslocation through the SEC61 translocon complex into the cytoplasmic compartment, where they are degraded by the proteasome.

on the genic etiology, although at least 1 case with a PKHD1 mutation (T-74) had macrocysts in the liver and 1 kidney cyst (Figure $5 \mathrm{~F})$. The pathogenicity of other uncommon missense variants in $P K H D 1$ is difficult to assess in the absence of robust functional assays. Seven probands not explained by any of the other PCLD gene mutations had somewhat rare $\left(<5 \times 10^{-3}\right.$ in ExAC) or novel missense variants in PKHD1 with combined annotation dependent depletion (CADD) scores greater than 14 (Supplemental Table 7). Four of the seven missense variants are predicted by MetaSVM (42), another aggregate prediction algorithm, to be deleterious (Supplemental Table 7). No comparably rare PKHD1 missense variants were found in any patient with mutations in the other known causative genes (SEC63, PRKCSH, ALG8, GANAB, or $S E C 61 B)$. In aggregate, mutations in $P K H D 1$ explain at least approximately $6 \%$ (10/159), and may explain up to approximately $11 \%$ (17/159), of clinically ascertained PCLD probands. While we did not find loss-of-function mutations nor any of the published pathogenic missense variants in LRP5 in our cohort, we did find 4 missense variants with MAF less than $1 \times 10^{-3}$ and CADD greater than 15 (Supplemental Table 8). All 4 of these variants occurred in cases that either were explained by loss-of-function mutations in PKHD1 (T-58 and T-59; Table 2) or may be explained by missense variants in PKHD1 (TOR6467 and TOR2400); Supplemental Tables 7 and 8). This does raise the possibility that genetic interactions with genes such as LRP5 may be a factor in determining the penetrance of PCLD in PKHD1 carriers.

PKHD1 is not known to belong to the ER protein biogenesis pathway defined by the other PCLD genes. We addressed this experimentally by inactivating $P k h d 1$ in $P k d 1^{F / H}-B A C$ cells. We produced 2 independent cell lines with either homozygous or compound heterozygous frameshift mutations in exon 3 of Pkhd1 (Sup-

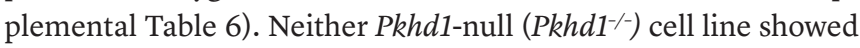
any changes in PC1-FL, PC1-CTF, PC1-NTR, or PC1-NTS levels when examined by anti-HA epitope or native protein $\mathrm{N}$-terminal antisera (Figure $5 G$ ). There was also no change in quantity of PC2 (Figure 5G). In keeping with the biochemical data, PC1 was readily detected in cilia of $P k h d 1^{-/-}$cells (Figure $5 \mathrm{H}$ ). The evidence suggests that $P K H D 1$ acts by a different mechanism than the protein biogenetic pathway impacted by the other PCLD genes. In aggregate, this study defines causative PCLD mutations in 4 genes that account for at least 20 probands in our discovery cohort of 102 and suggests that at least 2 different pathways are potential causes of clinically defined PCLD.

\section{Discussion}

Dominantly inherited mutations in SEC63 and PRKCSH cause PCLD by reducing the effective functioning dosage of PC1 in cells that undergo somatic second-hit mutations and result in recessive loss of function of the respective PCLD gene $(7,23)$. Once PC1 activity falls below a threshold, the rate of cyst progression is directly related to the degree of impairment of PC1 activity. This continuum also applies to $\operatorname{ADPKD}(43,44)$, but bile ducts appear to have a lower tolerance for reduced PC1 dosage compared with kidney tubules. This can be due to either a higher threshold requirement or a lower baseline level of PC1 activity in bile duct cells, which may explain the occurrence of PCLD rather than ADPKD with genetic defects affecting PC1 biogenesis. Identification of additional genes that impair PC1 functioning and manifest as human PCLD offers the opportunity to deepen our understanding of both ADPKD and PCLD. The advent of whole exome sequencing has enabled gene discovery that is not dependent on family data and is tolerant of diseases, such as PCLD, with extensive genetic heterogeneity (28-30). Applying this approach to studying the approximately $65 \%$ of clinically ascertained PCLD probands without mutations in SEC63 or $P R K C S H$, we found that no single gene explains more than $11 \%$ of the unknown cases. This suggests that there may be upwards of 10 to 15 causative genes for PCLD. 
Three genes identified in the current study, $A L G 8, G A N A B$, and $S E C 61 B$, fit into the mechanistic paradigm defined by SEC63 and PRKCSH. They encode proteins that function in the posttranslational protein biosynthetic pathways in the ER for integral membrane and secreted proteins (Figure 6). Analysis of in vitro cell models with knockout of each gene shows reduction in the steady-state levels of PC1 as a consistent feature. Beyond this common theme, there is substantial variation in the effects of each gene on PC1. Loss of Sec61b results in a substantially more severe deficiency of PC1 than loss of any of the other PCLD genes, including Sec63 and Prkcsh. Alg8 knockout causes hypoglycosylation of PC1, whereas Ganab knockout results in defective glucose trimming of N-glycan moieties that produces an increased apparent molecular mass for PC1. Inactivation of each of these 3 genes, including Ganab, results in activation of at least the IRE1 $\alpha / \mathrm{XBP} 1$ branch of UPR. This differs from Prkcsh, which does not activate UPR (33) despite the fact that the respective protein products of Prkcsh and Ganab work together as subunits of the GII holoenzyme. This suggests that the "cellular severity" of loss of the catalytic subunit, Ganab, is greater than that of the loss of the regulatory subunit, Prkcsh, required for ER retention. Whereas activation of XBP1s in Sec63 knockouts is required to support GPS cleavage of PC1 (33), we found that Xbp1s does not play this role in cells lacking Alg8.

Our finding that the pathogenic mechanisms of 5 PCLD genes converge on posttranslational modulation of PC1 function makes plausible the extended hypothesis that all genetic causes of PCLD occur by indirect impairment of effective PC1 signaling. This does not imply that all other PCLD genes affect PC1 posttranslational processing. Genetic defects that cause significant loss of PC1 activity by other indirect mechanisms may also result in PCLD. For example, PC1 acts as a negative regulator of an unidentified cilia-dependent signal that promotes cyst formation when intact cilia lack PC1 (45). Loss of function of any factor that works with PC1 in cilia as part of this negative regulatory activity may cause PCLD by impairing the efficiency of PC1 action.

PKHD1 and LRP5 are 2 examples of genes not clearly associated with PC1 biogenesis that are nonetheless associated with PCLD. We found genome-wide significant enrichment of heterozygous PKHD1 loss-of-function carriers in our cohort of clinically ascertained PCLD. This genetic discovery is consistent with a clinical report on the occurrence of innumerable asymptomatic liver cysts in approximately $10 \%$ of ARPKD carrier parents (46). Whether reduced or absent fibrocystin causes PCLD due to impaired PC1 signaling, or whether it causes PCLD independently of PC1, cannot be definitively answered with the available data. The former possibility is supported by strong evidence for a genetic interaction between $P k d 1$ and $P k h d 1$ in mouse models. Reduced $P k d 1$ dosage exacerbates the kidney and liver cysts in mice with homozygous Pkhd1 mutations $(7,24)$. This raises the possibility that somatic inactivation of PKHD1 may have a postdevelopmental role in bile ducts that sufficiently impairs effective signaling by PC1 in penetrant individuals to manifest with the sporadic cysts seen in PCLD.

$L R P 5$, a coreceptor for Frizzled in canonical Wnt signaling, has been proposed as a causal gene for PCLD (18). A recent study proposed that PC1 is a coreceptor for noncanonical Wnt signaling (47). These data may suggest an indirect functional relationship between LRP5 and PC1. We did not find any lossof-function mutations in LRP5, nor could we attribute any of the cases in our whole-exome-sequenced cohort to mutations in LRP5. However, we did note co-occurrence of indeterminate $L R P 5$ missense variants with PKHD1 variants. The phenotypes in these individuals did not stand out, but the co-occurrence of $L R P 5$ and $P K H D 1$ variants raises the possibility that incompletely penetrant heterozygous variants in $L R P 5$, and by extension other genes, may play a role in determining the penetrance of the PCLD phenotype in PKHD1 carriers.

Since the absolute number of PCLD patients with mutations in PKHD1 is small, it is not possible to draw definitive conclusions about clinical presentations. Nonetheless, some suggestive observations are worth noting. Five of the six PCLD patients explained by PKHD1 for whom imaging was available presented with a multitude of small cysts. This was also characteristic of the study that ascertained carrier parents of ARPKD children (46). This is in contrast to the larger cysts typical of most of the other PCLD genes and ADPKD. If fibrocystin is working through a PC1-dependent mechanism, a plausible hypothesis regarding this presentation is that the relative impairment of PC1 function due to loss of fibrocystin is less than that observed with loss of the other PCLD genes. Interestingly, the 2 cases of PCLD due to SEC61B mutations also presented with small cysts, yet SEC61B appears to have the most profound quantitative reduction in PC1 of all of the PCLD genes. This apparent paradox with the "PC1 dosage" hypothesis may be explained by the more severe and global effect of SEC61B inactivation on the rest of its non-PC1 client proteins. The drive toward tissue remodeling and cyst growth due to almost absent PC1 in bile duct epithelial cells lacking SEC61B may be partly counteracted by poor overall biosynthetic efficiency of other proteins required for cells to grow larger cysts. In future studies, clinically stratifying patients into those with macrocystic PCLD and those with consistently small cysts detected by MRI may enrich subsets of causative genes in these clinical subgroups.

$A L G 8$ presented the most pleiotropic clinical manifestations of PCLD and highlights that PCLD cyst growth may also be modified by additional genetic or nongenetic factors beyond the established association with female sex $(2,3)$. One male patient with $A L G 8$ mutation had severe PCLD that required procedural intervention to reduce liver cyst mass, while another had only minute cysts more typical of PKHD1 and SEC61B. Perhaps most interestingly, in a familial case attributed to $A L G 8$, the father had typical PCLD that included a few kidney cysts, while his daughter had 8 kidney cysts and no liver cysts at age 19. This suggests that $A L G 8$ may cross over clinically between PCLD and ADPKD. While this work was being prepared for publication, $G A N A B$ was independently identified as causing liver and kidney cysts in a population with a significantly greater burden of kidney cysts who were presumed to have ADPKD but who were excluded for mutations in PKD1 and PKD2 (48). The 2 novel $G A N A B$ patients in our study also have kidney cysts. Overall, it appears that $G A N A B$ and $A L G 8$ may straddle the diagnoses of PCLD and ADPKD, and mutations in $A L G 8$ may also explain some cases of apparent ADPKD that do not have mutations in $P K D 1$ or $P K D 2$ or GANAB. One mechanistic hypothesis for this multiorgan phenotype may be that GANAB and $A L G 8$ severe- 
ly affect PC1 maturation, but without the more global protein biosynthetic defects hypothesized for SEC61B that counter the cellular responses required for cyst growth.

PCLD due to $A L G 8$, like that due to PKHD1, occurs in adult carriers of a recessive disorder. ALG8 is the only one of the ERassociated PCLD genes for which a recessive genotype is reported to be compatible with life. Fifteen cases of congenital disorder of glycosylation $1 \mathrm{H}(\mathrm{CDG} 1 \mathrm{H})$ have been reported in the literature, caused by homozygous or compound heterozygous combinations of loss-of-function and deleterious missense mutations in ALG8 (49). The CDG1H phenotype has been reported to include renal failure and hepatomegaly with at least 1 report of bile duct dilation and bile duct cysts (50). Other features include protein-losing enteropathy, hypoalbuminemia, edema, ascites, and death in early infancy or childhood (51). Screening of $A L G 8$ heterozygous carrier parents by imaging may show an increased occurrence of PCLD much as was found in ARPKD carrier parents (46). This is the first instance of data supporting the hypothesis that PCLD may manifest in carriers of severe recessive diseases, particularly congenital disorders of glycosylation (50). Notably, recessive mutations in ALG9 cause CDG1L and are associated with polycystic kidneys $(52,53)$. Heterozygous carriers of $A L G 9$ mutations may manifest PCLD as well.

With the identification of 4 additional disease genes that can cause the PCLD phenotype, we have a genetic explanation of almost $50 \%$ of cases in our cohort of 159 probands. The newly identified ER genes confirm our previous discovery that defective PC1 biogenesis in the ER is central to the PCLD phenotype. This knowledge, the previous data that $P k d 1$ and $P k h d 1$ interact genetically, and our current finding that PKHD1 heterozygous carriers can also present with overlapping PCLD phenotypes provide increasing support for the hypothesis that the PC1 and fibrocystin signaling pathways intersect and each is sensitive to reduced dosage of the other. The co-occurrence of mild kidney cysts in some cases of PCLD supports the hypothesis that PCLD and ADP$\mathrm{KD}$ represent a clinical continuum that is partly a function of the severity of impairment of PC1 by the different genetic causes. The convergence on PC1 dependence of these phenotypes sets the dominant polycystic diseases apart from the recessive ciliopathy spectrum of disease. Discovery of other novel PCLD genes will identify additional potential modifiers of PC1 function. These genes may encode proteins functioning beyond the ER, perhaps even in cilia. These may prove to be powerful clues to unraveling the still-elusive function of the polycystin proteins.

\section{Methods}

Cohort recruitment and exome analysis. Index cases meeting entry criteria (see Results) for PCLD and at-risk family members were enrolled by study investigators at Yale, or sent to us deidentified by coinvestigators at other sites $(2,12,13)$. Representative liver and kidney imaging was obtained from the referring physician when available. Genomic DNA was obtained from the referring physician or extracted from peripheral blood leukocytes using Qiagen Puregene kit reagents. Genomic DNA from the proband was sent for targeted exome capture using the NimbleGen/Roche SeqCap EZ Exome v2 reagent, or MedExome reagent, followed by 75-bp paired-end sequencing using Illumina HiSeq 2000. Sequence reads were aligned to the GRCh37/ hg19 human reference genome using the Burrows Wheeler Aligner algorithm BWA-MEM (54). Variants were called using the Genome Analysis Toolkit Haplotype Caller (55). Annotations were carried out using scripts designed by the authors and members of the Yale Center for Genome Analysis. The analysis pipeline has been validated (29, 56) and makes use of SAMtools and Genome Analysis Toolkit utilities (55), and ANNOVAR software (57).

Bioinformatics. The observed gene burden of rare (ExAC frequency $<1 \times 10^{-3}$ ) loss-of-function alleles (nonsense, frameshift, splice site) was compared with the expected burden independent of ethnicity, and that in the European subset of discovery cohort cases $(n=92)$ was compared with that in European controls $(n=3,274)$ using existing scripts and statistical tests listed below. The European controls were composed of healthy parents of probands with autism and congenital heart disease sequenced using the same capture reagent, platform, and analysis pipeline as study cases. The $\mathrm{Q}-\mathrm{Q}$ plot was made using $\mathrm{R}$ software (https://www.r-project.org). PCA analysis used the EIGENSTRAT program (58). Pairwise kinship analysis was performed using PLINK (59). Identity-by-descent (IBD) analysis was performed using the phasing algorithm and IBD detection algorithm in the Beagle 4.1 software package $(60,61)$.

Statistics. $P$ values for observed versus expected gene burden comparisons were calculated using the binomial test, and for case versus control analysis using the Fisher's exact test. Statistical assessment of immunoblot densitometry data was done using paired-sample 2-tailed $t$ test.

In vitro gene knockout. All in vitro gene knockout studies were done in an immortalized mouse renal proximal tubule epithelial cell line containing 3 copies of a $P k d 1^{F / H}$ bacterial artificial chromosome (BAC) transgene (7). CRISPR/Cas9 was used to generate clonal cell lines containing protein-truncating mutations in the respective genes. Guide RNA sequences (Supplemental Table 6) were designed using the CRISPR Design tool (http://crispr.mit.edu/) (62) for Alg8, Xbp1, Pkhd1, and Sec61b, and using CRISPRscan (http://www.crisprscan.org) (63) for Ganab. For Alg8, Pkhd1, and Xbp1 knockouts, cells were infected with lentivirus containing lentiCRISPR v2 (Addgene plasmid 52961) (64). Following selection, clones were separated by clonal dilution, and screened by Sanger sequencing for frameshifting mutations. Ganab ${ }^{-/-}$ and $\mathrm{Sec} 61 \mathrm{~b}^{-/-}$cell lines were generated in a population of WT cells stably expressing Cas9 from lentiCas9-Blast (Addgene 52962). For each gene targeted, a large deletion was made between the sites of 2 guide RNAs in independent modified-pGL3-U6-sgRNA-PGK-puromycin (Addgene 51133) expression plasmids transiently transfected by electroporation. Clones were screened with PCR, then verified by Sanger sequencing. Re-expression of Alg8, Ganab, and Sec61b cDNA was done with lentivirus infection using the pCDH-CMV-MCS-EF1-Puro vector (System Biosciences) with a modified resistance cassette.

Immunoblotting. Cells were harvested and lysed in RIPA buffer containing Complete Protease Inhibitor Cocktail (Roche). Lysate was run on Novex 3\%-8\% Tris-acetate protein gels or Bio-Rad 10\% Tris-glycine protein gels, and transferred to PVDF membranes. Immunoblotting was performed using the primary antibodies listed below, and detected using Licor Odyssey Fc imaging system after application of HRP-conjugated secondary antibodies and washing with triethanolamine-buffered saline with Tween-20 (TBST).

Primary antibodies. The primary antibodies used were anti-HA (3F10, Roche; dilution 1:3,000), anti-PC1-NTF (7e12, Santa Cruz Biotechnology), anti-PC2 (YCC2, S. Somlo; 1:20,000), anti-HSP90 
(H114, Santa Cruz Biotechnology; 1:10,000), anti-GAPDH (14C10, Cell Signaling), anti-calnexin (ADI-SPA-860, Enzo), anti- $\alpha_{1}-\mathrm{Na}^{+} /$ $\mathrm{K}^{+}$-ATPase raised against chicken $\alpha_{1}-\mathrm{Na}^{+} / \mathrm{K}^{+}$-ATPase amino acids 338724 (a5, a gift of M.J. Caplan, Yale University, New Haven, Connecticut, USA) (65), anti-XBP1s (Poly6195, Biolegend; recognizes the C-terminal 15 amino acids of XBP1s), rabbit polyclonal anti-SEC61B (gift from R. Zimmerman, University of Saarland, Hamburg, Germany), anti-MYC (9e10, Santa Cruz Biotechnology), and anti- $\alpha$-glucosidase II (ab179805, Abcam; 1:2,000). All antibodies were used at 1:1,000 dilution in 5\% milk in TBST unless otherwise indicated.

Investigation of glycosylation status. Cell lysate was obtained in RIPA lysis buffer and incubated with anti-HA-conjugated agarose beads (Sigma-Aldrich). After washing, protein-bound beads were treated with EndoH or PNGase F (New England Biolabs). Treated PC1-HA protein was eluted from the beads by heating in $2 \mathrm{X}$ sample buffer (Sigma-Aldrich).

Immunofluorescence. Cells were cultured on glass coverslips to confluence and allowed to differentiate at $37^{\circ} \mathrm{C}$ without insulin/transferrin/selenium supplement (ITS), T3, or IFN- $\gamma$ for 10-14 days (7). They were serum-starved for 2 days to form cilia before analysis. Cells were fixed with $4 \%$ paraformaldehyde and permeabilized with $0.2 \%$ Triton before blocking ( $0.1 \%$ BSA plus $10 \%$ goat serum) and application of primary antibodies. After washing and fluorescent secondary antibody application, slides were imaged by confocal microscopy (Nikon) and analyzed using Elements software (Nikon).

Quantitative PCR and reverse transcription PCR. RNA was isolated from cultured cells using Trizol Reagent (Invitrogen). cDNA was reverse transcribed from RNA using reagents from New England Biolabs. Primers for Pkd1 (forward, ACACGCTTGGGAATGGACAC; reverse, CCATGGGAAGATGTTCTGGG) were designed using NCBI's primer designing tool (http://www.ncbi.nlm.nih.gov/tools/ primer-blast/). Quantitative PCR was done by Bio-Rad CFX Connect Real-Time PCR Detection System.

GII enzymatic testing. pCDNA3.1 eukaryotic expression plasmid expressing human GANAB cDNA sequence (gift of Anne Marcil, National Research Council, Ottawa, Ontario, Canada) was edited using site-directed mutagenesis methods. HEK 293T cells were cotransfected with pCDNA3.1 containing PRKCSH cDNA sequence and pCDNA3.1 containing GANAB sequence using Lipofectamine 2000 (Invitrogen). Lysate was made 72 hours after transfection using RIPA lysis buffer. Lysate was then incubated with a GII substrate, $1 \mathrm{mM}$ 4-methylumbelliferyl $\alpha$-D-glucopyranoside (4-MUG, Toronto Research Chemicals), at $37^{\circ} \mathrm{C}$ for 30 minutes, and reaction was stopped with $2 \mathrm{M}$ Tris. Fluorescence was measured with excitation/emission wavelengths
$365 / 445 \mathrm{~nm}$. Each transfection was done in triplicate, and each sample was tested with substrate in triplicate. Fluorescence values from empty vector-transfected cell lysate were subtracted from those of experimental samples to remove background native GII activity.

Study approval. This study was approved by the institutional review boards at both the Yale University School of Medicine and the Mayo Clinic. Written informed consent was obtained from all study participants prior to enrollment, and all samples and results were described and stored using a deidentified study code.

\section{Author contributions}

WB organized the cohort, carried out bioinformatics, designed and conducted experiments, and drafted the manuscript. KD and SVF developed cell lines and optimized experimental protocols. JC and MC conducted bioinformatics analysis. SP identified $A L G 8$ as a candidate gene and identified $P K H D 1$ variants. ARG provided reagents and experimental design for PKHD1 studies. JK and MC designed exome analysis pipelines and annotation scripts. $\mathrm{EBH}$ and AG performed Sanger sequencing verification of mutations. ET, PT, TW, YPP, and VET contributed patient samples and clinical information. SSC provided family studies. SM and RPL provided design of exome sequencing strategies. RPL designed bioinformatic evaluation. SS conceived and designed the project, organized collaborators, designed experiments, and edited the manuscript. Funding was obtained by WB and SS.

\section{Acknowledgments}

We gratefully acknowledge the generous participation of all the patients and families in this study. We thank Neera Dahl, Tamar Taddei, Simona Jakab, Joan Cho, James Boyer, Luca Fabris, Reiner Wiest, and David Roer for referring patients with PCLD. We thank Ali G. Gharavi for thoughtful discussions and critical reading of the manuscript, and Arnaud Marlier for contributing artwork. This study was supported by NIH grants R01 DK51041 and R01 DK54053 to SS; T32 DK007276 and a Polycystic Kidney Disease Foundation Fellowship to WB; the Yale Center for Mendelian Genomics (NIH U54 HG006504, to RPL); the Mayo Clinic PKD Center (P30 DK090728, to VET); and the Yale O'Brien Kidney Center (P30 DK079310).

Address correspondence to: Stefan Somlo, Section of Nephrology, Yale University School of Medicine, PO Box 208029, 333 Cedar Street, New Haven, Connecticut 06520-8029, USA. Phone: 203.737.2974; E-mail: stefan.somlo@yale.edu.
1. Reynolds DM, et al. Identification of a locus for autosomal dominant polycystic liver disease, on chromosome 19p13.2-13.1. Am J Hum Genet. 2000;67(6):1598-1604.

2. Qian Q, et al. Clinical profile of autosomal dominant polycystic liver disease. Hepatology. 2003;37(1):164-171.

3. Van Keimpema L, et al. Patients with isolated polycystic liver disease referred to liver centres: clinical characterization of 137 cases. Liver Int. 2011;31(1):92-98.

4. Pei Y, et al. Unified criteria for ultrasonographic diagnosis of ADPKD. JAm Soc Nephrol. 2009;20(1):205-212.
5. Desmet VJ. Ludwig symposium on biliary disorders - part I. Pathogenesis of ductal plate abnormalities. Mayo Clin Proc. 1998;73(1):80-89.

6. Karhunen PJ. Adult polycystic liver disease and biliary microhamartomas (von Meyenburg's complexes). Acta Pathol Microbiol Immunol Scand A. 1986;94(6):397-400.

7. Fedeles SV, et al. A genetic interaction network of five genes for human polycystic kidney and liver diseases defines polycystin-1 as the central determinant of cyst formation. Nat Genet. 2011;43(7):639-647.

8. Wills ES, Roepman R, Drenth JP. Polycystic liver disease: ductal plate malformation and the prima- ry cilium. Trends Mol Med. 2014;20(5):261-270.

9. Gabow PA, Johnson AM, Kaehny WD, MancoJohnson ML, Duley IT, Everson GT. Risk factors for the development of hepatic cysts in autosomal dominant polycystic kidney disease. Hepatology. 1990;11(6):1033-1037.

10. Chebib FT, et al. Outcomes and durability of hepatic reduction after combined partial hepatectomy and cyst fenestration for massive polycystic liver disease. JAm Coll Surg. 2016;223(1):118-126.e1.

11. Drenth JP, te Morsche RH, Smink R, Bonifacino JS, Jansen JB. Germline mutations in PRKCSH are associated with autosomal dominant polycystic liver disease. Nat Genet. 2003;33(3):345-347. 
12. Li A, et al. Mutations in PRKCSH cause isolated autosomal dominant polycystic liver disease. Am JHum Genet. 2003;72(3):691-703.

13. Davila S, et al. Mutations in SEC63 cause autosomal dominant polycystic liver disease. Nat Genet. 2004;36(6):575-577.

14. Yoder BK, Hou X, Guay-Woodford LM. The polycystic kidney disease proteins, polycystin-1, polycystin-2, polaris, and cystin, are co-localized in renal cilia. J Am Soc Nephrol. 2002;13(10):2508-2516.

15. Hilgendorf KI, Johnson CT, Jackson PK. The primary cilium as a cellular receiver: organizing ciliary GPCR signaling. Curr Opin Cell Biol. 2016;39:84-92.

16. Schou KB, Pedersen LB, Christensen ST. Ins and outs of GPCR signaling in primary cilia. EMBO Rep. 2015;16(9):1099-1113.

17. Nachury MV. How do cilia organize signalling cascades? Philos Trans R Soc Lond B Biol Sci. 2014;369(1650):20130465.

18. Cnossen WR, et al. Whole-exome sequencing reveals LRP5 mutations and canonical Wnt signaling associated with hepatic cystogenesis. Proc Natl Acad Sci U S A. 2014;111(14):5343-5348.

19. Qian F, Watnick TJ, Onuchic LF, Germino GG. The molecular basis of focal cyst formation in human autosomal dominant polycystic kidney disease type I. Cell. 1996;87(6):979-987.

20. Wu G, et al. Somatic inactivation of Pkd2 results in polycystic kidney disease. Cell.1998;93(2):177-188.

21. Janssen MJ, et al. Secondary, somatic mutations might promote cyst formation in patients with autosomal dominant polycystic liver disease. Gastroenterology. 2011;141(6):2056-2063.e2.

22. Watnick TJ, et al. Somatic mutation in individual liver cysts supports a two-hit model of cystogenesis in autosomal dominant polycystic kidney disease. Mol Cell. 1998;2(2):247-251.

23. Fedeles SV, Gallagher AR, Somlo S. Polycystin-1: a master regulator of intersecting cystic pathways. Trends Mol Med. 2014;20(5):251-260.

24. Garcia-Gonzalez MA, et al. Genetic interaction studies link autosomal dominant and recessive polycystic kidney disease in a common pathway. Hum Mol Genet. 2007;16(16):1940-1950.

25. Feldman M. Polycystic disease of the liver. AmJ Gastroenterol. 1958;29(1):83-86.

26. Karhunen PJ, Tenhu M. Adult polycystic liver and kidney diseases are separate entities. Clin Genet. 1986;30(1):29-37.

27. Melnick PJ. Polycystic liver; analysis of seventy cases. AMA Arch Pathol. 1955;59(2):162-172.

28. Stuart BD, et al. Exome sequencing links mutations in PARN and RTEL1 with familial pulmonary fibrosis and telomere shortening. Nat Genet. 2015;47(5):512-517.

29. Zaidi S, et al. De novo mutations in histone-modifying genes in congenital heart disease. Nature. 2013;498(7453):220-223.

30. Sanders SJ, et al. De novo mutations revealed by whole-exome sequencing are strongly associated with autism. Nature. 2012;485(7397):237-241.

31. Lek M, et al. Analysis of protein-coding genetic variation in 60,706 humans. Nature.
2016;536(7616):285-291.

32. Park E, Rapoport TA. Mechanisms of Sec61/ SecY-mediated protein translocation across membranes. Anпu Rev Biophys. 2012;41:21-40.

33. Fedeles SV, et al. Sec63 and Xbp1 regulate IRE1 $\alpha$ activity and polycystic disease severity. JClin Invest. 2015;125(5):1955-1967.

34. Qian F, et al. Cleavage of polycystin-1 requires the receptor for egg jelly domain and is disrupted by human autosomal-dominant polycystic kidney disease 1-associated mutations. Proc Natl Acad Sci US A. 2002;99(26):16981-16986.

35. Cai Y, et al. Altered trafficking and stability of polycystins underlie polycystic kidney disease. J Clin Invest. 2014;124(12):5129-5144.

36. Gainullin VG, Hopp K, Ward CJ, Hommerding CJ, Harris PC. Polycystin-1 maturation requires polycystin-2 in a dose-dependent manner. JClin Invest. 2015;125(2):607-620.

37. Kim H, et al. Ciliary membrane proteins traffic through the Golgi via a Rabep1/GGA1/Arl3-dependent mechanism. Nat Commun. 2014;5:5482.

38. Breitling J, Aebi M. N-linked protein glycosylation in the endoplasmic reticulum. Cold Spring Harb Perspect Biol. 2013;5(8):a013359.

39. Hetz C, Martinon F, Rodriguez D, Glimcher LH. The unfolded protein response: integrating stress signals through the stress sensor IRE1 $\alpha$. Physiol Rev. 2011;91(4):1219-1243.

40. Hofherr A, Wagner C, Fedeles S, Somlo S, Köttgen M. N-glycosylation determines the abundance of the transient receptor potential channel TRPP2. J Biol Chem. 2014;289(21):14854-14867.

41. Consugar MB, et al. Haplotype analysis improves molecular diagnostics of autosomal recessive polycystic kidney disease. Am J Kidney Dis. 2005;45(1):77-87.

42. Dong $\mathrm{C}$, et al. Comparison and integration of deleteriousness prediction methods for nonsynonymous SNVs in whole exome sequencing studies. Hum Mol Genet. 2015;24(8):2125-2137.

43. Cornec-Le Gall E, et al. Type of PKD1 mutation influences renal outcome in ADPKD. J Am Soc Nephrol. 2013;24(6):1006-1013.

44. Heyer CM, et al. Predicted mutation strength of nontruncating PKD1 mutations aids genotype-phenotype correlations in autosomal dominant polycystic kidney disease. J Am Soc Nephrol. 2016;27(9):2872-2884.

45. Ma M, Tian X, Igarashi P, Pazour GJ, Somlo S. Loss of cilia suppresses cyst growth in genetic models of autosomal dominant polycystic kidney disease. Nat Genet. 2013;45(9):1004-1012.

46. Gunay-Aygun M, et al. Hepatorenal findings in obligate heterozygotes for autosomal recessive polycystic kidney disease. Mol Genet Metab. 2011;104(4):677-681.

47. Kim S, et al. The polycystin complex mediates Wnt/Ca(2+) signalling. Nat Cell Biol. 2016;18(7):752-764.

48. Porath B, et al. Mutations in GANAB, encoding the glucosidase II $\alpha$ subunit, cause autosomaldominant polycystic kidney and liver disease. Am J Hum Genet. 2016;98(6):1193-1207.

49. Höck M, et al. ALG8-CDG: novel patients and review of the literature. Orphanet J Rare Dis. 2015;10:73.

50. Janssen MJ, Waanders E, Woudenberg J, Lefeber DJ, Drenth JP. Congenital disorders of glycosylation in hepatology: the example of polycystic liver disease. J Hepatol. 2010;52(3):432-440.

51. Freeze HH. Genetic defects in the human glycome. Nat Rev Genet. 2006;7(7):537-551.

52. Weinstein M, et al. CDG-IL: an infant with a novel mutation in the ALG9 gene and additional phenotypic features. Am JMed Genet $A$. 2005;136(2):194-197.

53. Tham E, et al. A novel phenotype in N-glycosylation disorders: Gillessen-Kaesbach-Nishimura skeletal dysplasia due to pathogenic variants in ALG9. Eur J Hum Genet. 2016;24(2):198-207.

54. Li H, Durbin R. Fast and accurate short read alignment with Burrows-Wheeler transform. Bioinformatics. 2009;25(14):1754-1760.

55. McKenna A, et al. The Genome Analysis Toolkit: a MapReduce framework for analyzing nextgeneration DNA sequencing data. Genome Res. 2010;20(9):1297-1303.

56. Choi M, et al. Genetic diagnosis by whole exome capture and massively parallel DNA sequencing. Proc Natl Acad Sci U S A. 2009;106(45):19096-19101.

57. Wang K, Li M, Hakonarson H. ANNOVAR: functional annotation of genetic variants from high-throughput sequencing data. Nucleic Acids Res. 2010;38(16):e164.

58. Price AL, Patterson NJ, Plenge RM, Weinblatt ME, Shadick NA, Reich D. Principal components analysis corrects for stratification in genome-wide association studies. Nat Genet. 2006;38(8):904-909.

59. Purcell S, et al. PLINK: a tool set for whole-genome association and population-based linkage analyses. Am JHum Genet. 2007;81(3):559-575.

60. Browning BL, Browning SR. Improving the accuracy and efficiency of identity-bydescent detection in population data. Genetics. 2013;194(2):459-471.

61. Browning SR, Browning BL. Rapid and accurate haplotype phasing and missing-data inference for whole-genome association studies by use of localized haplotype clustering. Am J Hum Genet. 2007;81(5):1084-1097.

62. Hsu PD, et al. DNA targeting specificity of RNA-guided Cas9 nucleases. Nat Biotechnol. 2013;31(9):827-832.

63. Moreno-Mateos MA, et al. CRISPRscan: designing highly efficient sgRNAs for CRISPR-Cas 9 targeting in vivo. Nat Methods. 2015;12(10):982-988.

64. Sanjana NE, Shalem O, Zhang F. Improved vectors and genome-wide libraries for CRISPR screening. Nat Methods. 2014;11(8):783-784.

65. Tamkun MM, Fambrough DM. The (Na++ $\mathrm{K}+$ )-ATPase of chick sensory neurons. Studies on biosynthesis and intracellular transport. J Biol Chem. 1986;261(3):1009-1019.

66. Xu H, Shen J, Walker CL, Kleymenova E. Tissue-specific expression and splicing of the rat polycystic kidney disease 1 gene. DNA Seq. 2001;12(5-6):361-366. 